\title{
Pigeons and People: resource ecology and human dimensions of urban wildlife
}

\author{
by \\ Symone Krimowa
}

\author{
A thesis \\ submitted to the Victoria University of Wellington \\ in partial fulfilment of the \\ requirements for the degree of \\ Master of Science \\ in Conservation Biology. \\ Victoria University of Wellington
}

2012 



\begin{abstract}
Urban areas and human populations are growing. Cities provide highly modified habitat for species that can adapt their feeding and other behaviours. The growth of urban landscapes and human populations may result in an increase in human-wildlife conflict. Businesses which prepare and sell food (food establishments) may be more likely to encounter conflict with urban wildlife, which may lead to negative attitudes towards urban wildlife. Negative attitudes towards wildlife could create polarised communities and possibly affect the success of environmental initiatives.

This study sought to understand (1) how feral pigeons use urban environments and the resources key to their distribution and congregation; (2) whether feral pigeons are food limited in Wellington City; and (3) how the interactions of owners and managers of food establishments with feral pigeons influence their attitudes to feral pigeons.

I used 8 transects through the central City which covered a representative sample of urban habitats, including the central business district, green space, and waterfront to estimate resource selection. Bird capture and banding were used to determine feral pigeon condition at a range of sites across the City and included a mix of high, medium and low anthropogenic fed sites. A written survey of owners and managers of food
\end{abstract}


establishments in Wellington was conducted to evaluate attitudes to feral pigeons $(n=62)$.

Feral pigeon resource selection is mainly influenced by people and where they choose to eat $(\triangle A I C \leq W=0.999)$, such as sites with outdoor seating where people may directly feed feral pigeons. However, once a site has been selected, areas with tertiary vegetation and disposed food ( $W=0.324$ and $W=0.297)$ are the most likely to attract larger flocks of feral pigeons (although a number of other variables also influence flock size, such as availability of freshwater).

Feral pigeons do not appear to be food limited in Wellington as condition was not significantly different between sites $(n=48$, body condition, (body mass /tarsus length) Kruskal-Wallis $=2.06, \mathrm{p}=0.36$; keel condition, Kruskal-Wallis $=0.7283, \mathrm{p}=0.6948$; feather condition Kruskal-Wallis $=$ $2.7943, \mathrm{p}=0.2473)$.

Attitudes of food establishment owners and managers towards feral pigeons are most influenced by how often they see feral pigeons ( $\triangle A I C c \leq$ $W=0.465)$. Therefore, direct experience rather than knowledge, engagement, action or socio-demographics has the most influence on attitudes of owners and managers of food establishments.

These results suggest that feral pigeon populations are largely dependent on the availability of anthropogenic foods. Reducing the food provided by people may limit feral pigeon populations. Reductions in pigeon populations are also likely to change attitudes of business owners and reduce conflict because they will be less likely to encounter pigeons. Limit- 
ing feeding and access to food waste is probably the most effective way of managing pigeon populations. 


\section{Acknowledgments}

I would like to thank my supervisor, Wayne Linklater, for his support and contribution to this thesis - you always made time to discuss and read through my endless drafts. I would also like to acknowledge Monica Awasthy for her valuable assistance with all the early morning fieldwork in the City - I could not have done it without you. I am very grateful to Alice Ryan for being an excellent field work partner and friend over the last few years - the thesis was a lot more fun because of your input.

The support from my partner and family has been invaluable throughout and you cannot be thanked enough. 


\section{Contents}

1 Introduction 1

1.0.1 Human-wildlife relationships . . . . . . . . . 3

1.0.2 Management ................ 5

1.0 .3 Study site .................. 7

1.0.4 Study objectives . . . . . . . . . . . 8

2 Resource selection $\quad 11$

2.1 Introduction . . . . . . . . . . . . . . . 11

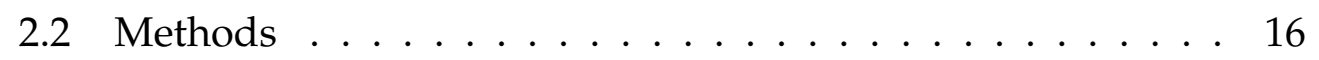

2.2.1 Study site and animal . . . . . . . . . 16

2.2 .2 Sampling ..................... 17

2.2 .3 Fixed-effects . . . . . . . . . . . . . . 19

2.2.4 Candidate models . . . . . . . . . . . . . 20

2.2 .5 Statistical method . . . . . . . . . . . . . 21

2.3 Results ........................ 22

2.3 .1 Site Selection . . . . . . . . . . . . . 22

2.3 .2 Flock size . . . . . . . . . . . . . 23

vii 
2.4 Discussion . . . . . . . . . . . . . . 27

2.4 .1 Flock size . . . . . . . . . . . . 32

2.5 Management implications . . . . . . . . . . . . 33

3 Anthropogenic food and urban wildlife 37

3.1 Introduction . . . . . . . . . . . . . 37

3.2 Methods ...................... 40

3.2.1 Study site and population . . . . . . . . . . 40

3.2.2 Experimental design . . . . . . . . . . . 40

3.2.3 Statistical method . . . . . . . . . . . . . 42

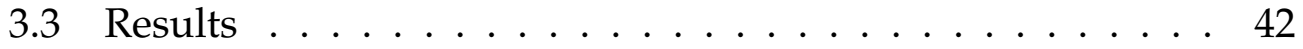

3.4 Discussion ....................... 48

4 Business attitudes $\quad 53$

4.1 Introduction . . . . . . . . . . . . . 53

4.2 Methods ...................... 56

4.2.1 Study area and population . . . . . . . . 56

4.2 .2 Sampling . . . . . . . . . . . . 57

4.2.3 Questionnaire design .............. 58

4.2.4 Candidate models . . . . . . . . . . . . . . 60

4.2 .5 Statistical method . . . . . . . . . . . 61

4.3 Results ...................... 63

4.3.1 Model Results . . . . . . . . . . . . . . . 64

4.4 Discussion ...................... 73

4.4 .1 Attitudes ................... 74 
CONTENTS ix

5 Conclusion $\quad 79$

5.1 Management implications . . . . . . . . . . . . 83

$\begin{array}{lr}\text { A Appendices } & 89\end{array}$ 


\section{List of Figures}

2.1 The proportion of sites that were selected and available in central Wellington City for cafés, outdoor seating, squares and grassed areas $(n=193)$. The sites are from the best performing model ( $\triangle \mathrm{AIC} \leq 2, W=0.999)$, which suggests that areas where people congregate to eat is the primary factor influencing feral pigeon site selection. The survey consisted of eight line transects through central Wellington and was completed in summer (February - March) and winter (July - August) over a 2-3 week period. . . . . . . . . . . . . . . 25 
3.1 Distribution of results of feral pigeon condition (body weight/tarsus) across three sites in Wellington: low $(n=12)$, medium $(n=18)$ and high $(n=18)$ fed. Each site was categorised as low, medium or high fed by observation of the amount of anthropogenic food seen between June and October 2010. Birds were banded $(n=48)$ at each site using mist and hand nets in winter 2010. Body weight was measured using a scale. The box and whisker plots indicate the average by the + symbol and the median by the line in the middle of the box. The boxes show the 25th quartile and the 75th quartile with extensions showing the range of values. . . . . 44

3.2 Distribution of results of adult feral pigeon keel condition across three sites in Wellington: low $(n=12)$, medium $(n=18)$ and high $(n=18)$ fed. Each site was categorised as low, medium or high fed by observation of the amount of anthropogenic food seen between June and October 2010. Birds were banded at each site using mist and hand nets in winter 2010. Keel condition was estimated by the amount of fat felt with two fingers. The box and whisker plots indicate the average by the + symbol and the median by the line in the middle of the box. The boxes show the 25th quartile and the 75th quartile with extensions showing the range of values. 45 
3.3 Distribution of results of feral pigeon feather condition across three sites in Wellington: low $(n=12)$, medium $(n=18)$ and high $(n=18)$ fed. Each site was categorised as low, medium or high fed by observation of the amount of anthropogenic food seen between June and October 2010.Birds were banded and measured at each site using mist and hand nets in winter 2010. The box and whisker plots indicates the average by the + symbol. The boxes show the 25 th quartile and the 75th quartile with extensions showing the range of values. . . . . . . . . . . . . . . 46

3.4 Frequency of feral pigeon activity across three sites in Wellington: low, medium and high fed. The level of feeding at each site was quantified by observation into low, medium and high fed. Birds were banded and measured at each site using mist and hand nets in winter $2010(n=48)$. Activities (resting or foraging) of feral pigeons throughout Wellington were recorded during winter and spring $2010(n=285)$. . . . 47

4.1 Attitude of respondents to feral pigeons and the annual cost of feral pigeons to food establishments $(n=62) \ldots \ldots 65$

4.2 Attitude of respondents to feral pigeons and the frequency feral pigeons were seen at food establishments $(n=62)$. . . 66

4.3 Attitude of respondents to feral pigeons and the frequency feral pigeons obtain or receive food from establishments ( $n=$

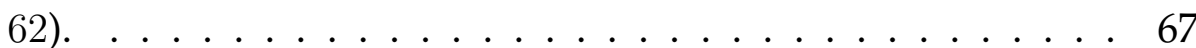


4.4 Attitude of respondents to feral pigeons on number of pigeons by age of respondents $(n=62) \ldots \ldots \ldots$

4.5 Attitude of respondents to feral pigeons by sex of respon-

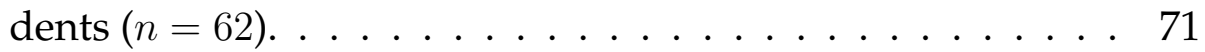

4.6 Attitude of respondents to feral pigeons by education level of respondents $(n=62) \ldots \ldots \ldots \ldots$. . . . . . . . . . . 


\section{List of Tables}

2.1 Feral pigeon group resource selection . . . . . . . . . . 24

2.2 Larger group selection of resources . . . . . . . . . . . . 26

4.1 Candidate models with socio-demographic variables (age, sex and education variables are indicated by $\left.{ }^{* * *}\right)$. . . . . . 69

4.2 Attitudes of food establishments towards feral pigeons . . . 70 


\section{Chapter 1}

\section{Introduction}

By 2050 69\% of the world's human population will live in cities (UnitedNations, 2009). In New Zealand, 75\% of people live in urban areas (Statistics, 2006). Similarly, in other developed countries most people already live in urban centres. As a result, landscapes are being transformed from rural to urban. Urban areas are places of dense human population, where most of the land is dedicated to impervious surfaces (e.g., buildings and pavement), with isolated green space (Adams et al., 2006). Cities provide highly modified habitat for some species of wildlife. Urban ecosystems are usually characterised by high food abundance (Anderies et al., 2007), low predation risk (Tsurim et al., 2008), permanent water sources and shelter from harsh climates (Rebele, 1994). Compared with wildlands, cities are characterised by higher bird population densities, but lower species richness (Shochat et al., 2004).

Adaption to urban ecosystems requires changes in the behaviour and 
ecology of birds. Birds must change feeding behaviours, food types and nesting habits, e.g., using anthropogenic objects as shelters, nesting places and materials for nests (Luniak, 2004). Wildlife living amongst people often display different resource preferences than their counterparts living in less modified or wild habitats. There is a lack of understanding about how exotic species exist so successfully in highly modified urban environments.

One of the most successful colonisers of urban environments is the feral pigeon (Columba livia) (Haag-Wackernagel et al., 2006). Urban centres across the world are home to feral pigeons who substitute their original cliff-dwelling habitat for city buildings. Feral pigeons nest and roost in tall buildings and forage in parks and city squares (Johnston and Janiga, 1995). The feral pigeon has become widely established in New Zealand (Dilks, 1975), particularly in the centres of major cities, following introduction by early Europeans (Robertson et al., 2007).

Compared to the domestic variety, feral pigeons fly faster, have higher annual reproductive potential (can breed year-round), and greater genetic variability (Adams et al., 2006). Feral pigeons have adapted their original diets of grain to include anthropogenic sources of food. Urban feral pigeons may depend on the public for food sources or steal food from grain storage areas, e.g., bakeries (Murton et al., 1972b). 


\subsubsection{Human-wildlife relationships}

The most important difference between urban and less modified or wild ecosystems is the major role humans play. Beyond being the dominant urban species, people influence species composition and abundance by killing pests, and feeding and protecting popular species, particularly birds (Tangley, 1986). Indeed, studies have indicated that up to $57 \%$ of people may feed wildlife (Rollinson et al., 2003), which can influence population size.

Urban wildlife and humans' interests can conflict and the frequency and severity of these conflicts has increased (Madden, 2004). These conflicts can influence people's attitudes towards wildlife and result in polarised views within the urban community (Temby, 2004). In particular, people at the receiving end of wildlife damage tend to oppose conservation agendas (Sillero-Zubiri et al., 2007).

Conflict with wildlife in urban areas is common and can range from large urban fox populations in Great Britain to fouling by pigeons, which can be politically difficult for cities to manage, e.g., London's mayor's political problems over pigeon management in high profile city squares. Feral pigeons have a long history of human-wildlife conflict, which includes unwanted noise to urban residents (Jerolmack, 2008), the damage feral pigeons cause to buildings and other surfaces from nesting and feces (Sacchi et al., 2002), and the potential of feral pigeons to spread human diseases (Haag-Wackernagel and Moch, 2004). An individual pigeon produces around $12 \mathrm{~kg}$ of excrement per year that fouls breeding ar- 
eas, building faces, monuments, pavements and other public areas (HaagWackernagel and Geigenfeind, 2008). Feral pigeons harbour at least 110 different human pathogens, but there has been limited evidence of actual human infection with only 230 recorded human infections worldwide (Haag-Wackernagel and Moch, 2004). While others view pigeons as complementary to city life and enjoy feeding and interacting pigeons (Johnston and Janiga, 1995). Those who enjoy pigeons in cities are likely to hold strong opinions against control — leading to human-wildlife conflict.

In the urban context, people are increasingly removed from interactions both with wildlife and domesticated animals used in food production (Patterson et al., 2003). Most human contact with animals is with pets. Therefore, people can tend to perceive urban wildlife similarly to pets, which is demonstrated by how some people regularly feed pigeons and other wild birds. Those in the urban community who bear the costs of urban wildlife, such as damage to buildings, are likely to have different attitudes toward wildlife than those in the community who view wildlife similarly to pets. Thus, human-wildlife conflict frequently involves an equally important conflict between people who have different goals, attitudes, levels of empowerment and wealth (Madden, 2004). The challenge of addressing the conflict is understanding the human dimension with its social, cultural, political, economic and legal complexities (Madden, 2004).

To address urban wildlife conflict, both the scientific understanding of urban wildlife as well as people's perceptions of the conflicts are critical to managing the conflicts (Sillero-Zubiri et al., 2007). People's atti- 
tudes are influenced by socio-demographics (e.g., age, sex, ethnicity) and and a wide variety of other dimensions, such as knowledge, direct experience, values, beliefs and affiliations (Cordano et al., 2004) as well as place attachment (Vorkinn and Riese, 2001). Some research has demonstrated that behavior is more accurately predicted from attitudes formed via direct, behavioral interaction with the attitude object (e.g., wildlife) than from attitudes developed via indirect, nonbehavioural experience (Fazio and Zanna, 1981). Attitudes formed on the basis of direct experience are more predictive of behaviour than attitudes formed by indirect experience (Fazio, 1986). So people's experience of wildlife may be formative to positive and negative attitudes with implications for managing human-wildlife conflict.

There is a large body of literature available on feral pigeons in countries around the world, particularly in Europe, but the research on feral pigeons in New Zealand has been limited to feral pigeons in rural agricultural areas (Dilks, 1975). Therefore, it is unknown how feral pigeons exist in urban areas in New Zealand. This thesis aims to improve urban wildlife management through a reduction in human-wildlife conflict by understanding how feral pigeons exist in highly modified urban habitats and how they affect people's attitudes.

\subsubsection{Management}

Feral pigeons in urban areas create a human-wildlife conflict that city authorities struggle to manage. Population management of feral pigeons 
may ease the conflict between people and pigeons. The public's concerns over feral pigeons needs to be addressed because research has indicated that people's associations with wildlife may have important consequences for their willingness to participate in wildlife conservation (Dunn et al., 2006). If people who live in urban centres only interact with urban wildlife species such as feral pigeons with which there is conflict, they may form negative views towards other wildlife (Dunn et al., 2006). Alternatively, people may be more likely to form positive views of the wrong type of wildlife, for example, pest species. And, people may therefore have little interest in participating in agendas focused on wildlife conservation.

City authorities are familiar with some people's opposition to population management through culling. Previous works indicate that residents retain positive attitudes towards even pest wildlife in urban centres. Feral pigeon management may receive more positive publicity if done in a manner that conforms with public opinion and animal welfare concerns (Reiter et al., 1999). Population control is also necessary for the protection of pigeons from the public's attempts to "carry out their own pigeon management" (DominionPost, 2008) which may not comply with animal welfare standards. In New Zealand, feral pigeon population management must comply with the Wildlife Act of 1953 and the Animal Welfare Act of 1999. The general public is more likely to support wildlife management methods that are non-lethal (Reiter et al., 1999).

Culling is not always the best solution because it is very costly and population levels return to previous numbers very quickly through immigra- 
tion/recruitment. There are also logistical problems with humans nearby in urban areas (e.g., safety concerns with poison and shooting) (Murton et al., 1972a). For example, the latest Wellington City Council campaign to cull pigeons was cancelled due to an assessment by Council staff that culling will not effectively reduce pigeon numbers (DominionPost, 2008). The cost of population control by continuous culling was estimated by the Council to be up to $\$ 100,000$ per year (DominionPost, 2008) which the Council considered to be too expensive for pigeon population control.

Population management of feral pigeons might be better achieved by the modification or reduction of resources available to feral pigeons in the central city. Research has shown that non-lethal methods, such as food supply reduction, may be the best method to control feral pigeon populations in urban areas (Murton et al., 1972a) because of its long-term effectiveness and limited public opposition.

\subsubsection{Study site}

The study took place in central Wellington City. Wellington City is New Zealand's third most populous city with 195,000 people. The City is located on the edge of a harbour and is surrounded by steep hills and has a mild temperate, oceanic climate. It is well-known for its strong northerly and southerly winds and hilly topography. The climate is sunnier than some other cities with feral pigeons with over 2,000 hours of sunshine a year. The coldest month is July with an average temperature of 8.7 degrees Celsius and the warmest month is February with an average temperature 
of 17 degrees Celsius. Average rainfall is 1,270 mm.

Wellington is an interesting city in which to study feral pigeons because, unlike in other cities, Wellington is not surrounded by intensive agriculture, such as mixed cropping. Consequently, pigeons are unlikely to depend on leaving the city to feed in agricultural fields for the day and return at night to roost. In areas outside of Europe such as Canada, India, and New Zealand, feral pigeons do not readily adjust to eating seeds of native plants (Johnston and Janiga, 1995). Therefore, feral pigeons in Wellington City may rely solely on anthropogenic food.

Feral pigeons are resident year-round in Wellington City and are capable of breeding year-round in New Zealand (Dilks, 1975). The year-round breeding pattern is similar to many other countries (Schein, 1954) - even those with much harsher climates. The continuous breeding cycle is likely due to lack of seasonal variation of anthropogenic food supply in urban centres (Johnston and Janiga, 1995).

\subsubsection{Study objectives}

The objectives of this study are to understand how feral pigeons use urban environments and the resources key to their distribution and congregation - particularly by understanding what influences feral pigeon site choice and aggregation. As a commensal species, it is important to understand what resources are supporting pigeon populations and larger flocks of feral pigeons, which are a key problem in urban areas.

This study also aims to determine whether feral pigeons in Wellington 
City are food limited. By investigating whether the quantity of feeding by people at central Wellington sites effects feral pigeon condition and activity, we can determine whether food supply by people may limit Wellington City's pigeon population.

Finally, the study also seeks to understand how owners and managers of food establishments' interactions with feral pigeons influence their attitudes towards feral pigeons. Particular attention will given to whether direct behavioural experience influences attitudes towards feral pigeons. By understanding what is driving peoples' attitudes towards feral pigeons, we can begin to manage human-wildlife conflict in urban areas.

Each chapter is written as a stand-alone manuscript for journal submission and some repetition may occur in the descriptions of study site and study species. 


\section{Chapter 2}

\section{Resource selection by feral}

pigeons in an urban environment: the importance of people and anthropogenic food

\subsection{Introduction}

Urban environments will have more than half the world's population within the next 25 years. Urban areas provide highly modified habitat for some wildlife, particularly commensal species (species that have a close association with humans) (Clark, 2011). The urban ecosystem is usually characterised by high food resource abundance (Anderies et al., 2007), low predation risk (Tsurim et al., 2008), supplementary feeding by humans 
(Major et al., 1996) permanent water sources and shelter from harsh climates (Rebele, 1994) allowing commensal species to reach high densities and aggregate in large numbers. Urban areas are places of dense human population, where most of the land is dedicated to buildings and concrete, with isolated green space (Adams et al., 2006). Compared with adjacent wildlands, cities are characterised by higher bird population densities, but lower species richness (Shochat et al., 2004).

Birds in urban ecosystems have adapted their behaviour and ecology from those living in less modified habitats. Urban birds must change feeding behaviours and nesting habits, e.g., using anthropogenic objects as shelters, nesting places and nest materials (Luniak, 2004). Commensal species often display different resource preferences than the same species living in non-urban areas. There is a lack of understanding about how commensal species exist in such high numbers in highly modified urban environments.

One of the most successful colonisers of urban environments is the feral pigeon (Columba livia) (Haag-Wackernagel et al., 2006). Compared to the domestic variety, feral pigeons fly faster, have a higher annual reproductive potential, and greater genetic variability (Adams et al., 2006). Feral pigeons have adapted their original diets of grain to include anthropogenic sources of food. Urban feral pigeons may depend on the public for food sources or steal food from cafes (Murton et al., 1972b). In areas where agricultural fields are in close proximity to urban centres, feral pigeons may use the city for nesting and roosting but travel outside the city to feed on 
a daily basis (Johnston and Janiga, 1995). In some studies, feral pigeons choose urban feeding sites within the city (Jokimäki and Suhonen, 1998).

Feral pigeons usually feed twice a day, at least in winter (Johnston and Janiga, 1995). Feral pigeons living in urban areas, without access to traditional seeds and food sources, have successfully adapted to an omnivorous diet that is built upon their original diet of grains (Ciminari et al., 2005). In some cities feral pigeons rely solely on the food provided by people (Sacchi et al., 2002). Bread, cakes, household food scraps, and cereals provided by the public make up a substantial proportion of urban feral pigeons' diets (Murton and Westwood, 1966). In urban areas, such as the site of this study, feral pigeons may be limited to human sources of food. Unlike in other cities, the city of this study is not surrounded by intensive agriculture, such as mixed cropping. Therefore, pigeons may be unlikely to leave the city to feed in agricultural fields for the day and return at night to nest. In areas outside of Europe such as Canada, India, and New Zealand, feral pigeons do not readily adjust to eating seeds of native plants (Johnston and Janiga, 1995).

Water is essential to feral pigeons, particularly during breeding for the production of crop milk (a mixture of seeds and water). Pigeons are unique among bird species for their ability to drink without lifting their heads. The pigeons' beak is similar to a straw that is able to suck water from scant sources, such as puddles.

Feral pigeons have also readily adapted to urban infrastructure in place of traditional cliff-dwelling locations to use as roosting and nesting spots. 
Feral pigeons' favoured nesting spots are high-up in gaps and openings of buildings, parking garages, bridges, and beneath the wharves along the waterfront. They prefer sites that are sheltered from the wind.

Urban birds face different types of stresses compared to wild birds. For example, urban feral pigeons do not face predation pressure from possums like other New Zealand birds. Urban stresses to feral pigeons include, traffic, noise (Warren et al., 2006), pollution (Nam et al., 2004), and, in some areas, high densities of other predators, such as hawks (Sorace, 2002). There are usually lower numbers of native predators in urban environments and higher densities of domestic and feral predators, such as cats (Shochat et al., 2004). However, despite the low number of native predators, some birds may perceive urbanised habitats as more dangerous because of the presence of human activities. Human activities can increase the risk of predation or modify the ability to detect predators (Valcarcel and Fernández-Juricic, 2009), which can increase stress levels.

Urban stresses, such as human activity, can influence reproductive characteristics such as timing of breeding, clutch size, and mate acquisition (Ditchkoff et al., 2006). Despite high stress induced by a range of factors associated with urban life, urban habitats throughout the world are characterised by high population densities of certain species (Anderies et al., 2007). In Wellington City, the most likely threats to feral pigeons are people and traffic. Although, in more residential or suburban areas of Wellington, feral pigeons may be at risk of predation from cats and hawks.

The aim of this study is to understand what influences feral pigeon 
site choice and aggregation. Special attention will be given to what drives larger groups of feral pigeons to thrive in cities. Larger groups of feral pigeons are more likely to cause damage and disturb people. Our study objectives were to (1) understand feral pigeon habitat selection in Wellington City; and (2) determine large groups of feral pigeons' preferences for certain resources. This study is the first in New Zealand to analyse feral pigeon resource use in urban environments. 


\subsection{Methods}

\subsubsection{Study site and animal}

The study was completed in central-Wellington City. Wellington City is New Zealand's third most populous city with 195,000 residents. Wellington City is located within around a harbour and is surrounded by steep hill country. It has a mild temperate oceanic climate and is well-known for its strong winds (predominantly northerly). Wind is especially significant for feral pigeons in Wellington City because of the high annual wind speeds (mean annual wind speed of $22 \mathrm{~km} / \mathrm{hr}$, with 173 days having gusts of over $60 \mathrm{~km} / \mathrm{hr}$ ), which can limit reproductive success (Johnston and Janiga, 1995). The climate is sunnier than some other cities with feral pigeons with over 2,000 hours of sunshine a year. The coldest month is July with an average temperature of 8.7 degrees Celsius and the warmest month is February with an average temperature of 17 degrees Celsius. Average rainfall is $1,270 \mathrm{~mm}$. The city's architecture is a mixture of wood and concrete buildings build during the last 150 years. There are a high number of vegetated (green) spaces, including native forest, exotic pine forest and open space, surrounding the city. The town belt extends for 425 ha through the city and there are many urban parks. Notably different than many other cities in New Zealand is the lack of intensively developed agricultural and horticultural land near the city. As a result, pigeons are less likely to leave the city to feed during the day. A variety of habitats are available across the study area including, wharves and waterfront, urban 
parks and gardens, residential areas and the central business district.

It is common to see feral pigeons year-round in Wellington City. The species is capable of breeding year-round in New Zealand and shows only slight seasonal breeding peaks (Dilks, 1975). This breeding pattern is similar in many other countries (Schein, 1954) - even those with much harsher climates. The continuous breeding cycle is likely due to lack of seasonal variation in food supply in urban centres where anthropogenic foods form most of the diet. Research suggests there is little habitat variation between seasons with the same amount of food available throughout the year (Murton et al., 1972a).

There is a large body of literature available on feral pigeons in countries around the world, particularly in Europe, but the research on feral pigeons in New Zealand has been limited to feral pigeons in rural agricultural areas (Dilks, 1975). Therefore, it is unknown how feral pigeons exist in urban areas in New Zealand.

\subsubsection{Sampling}

Line transects across central Wellington City were used to measure feral pigeon resource selection. The transects covered a representative sample of urban habitats. The urban habitat of Wellington City includes green space, central business district, wharves and public squares.

Each of the eight transects began in the northeast and proceeded southwest across the city. Direction was determined using a Casio digital compass (Casio Pathfinder PAG-40). The transects were evenly spaced apart 
and were drawn in a zig-zag pattern beginning at a random point on the study boundary. Each transect followed roads, alleys, and paths within a defined set of criteria including, alternating right and left turns at intersections to follow a northeast-southwest direction. We also forced the transect direction to comply with the northeast-southwest direction, i.e., when following the "turn right, then left" criteria led the route away from the northeast-southwest direction. The transects were surveyed in summer (February - March) and winter (July - August) 2010 over a 2-3 week period.

Available resources were measured to determine their selection (Manly, 2002) by groups of feral pigeons that varied in size. Habitat preference is inferred through the disproportionate use of some resources over others (Neu et al., 1974). When one habitat is used less, others must be used more (Johnson, 1980). The same transects were used to measure resource selection and availability. Available resources were measured by recording resources available at distinct points along each transect. Resources were measured every 200 meters along the entire length of each transect. At each distinct point resources available at ground level, 10 meters, 30 meters, 50 meters, and 70 meters elevation were recorded so that all potential resources used by pigeons were included. The 200 meter distance between each point was measured using a global positioning system (GPS) hand held unit. And, the distance to each resource level was measured using a range finder.

The data from both summer and winter seasons was combined so that 
analyses of feral pigeon resource selection could focus on an annual pattern.

The response variables measured were the presence of feral pigeon groups and the number of feral pigeons in each group. A group of feral pigeons was defined by estimating the greatest distance between birds in a prospective group. If the distance between any two pigeons was greater than the distance between the furthest remaining birds of the group, then that individual was identified as separate from the group. A group could be as small as one feral pigeon. Sites with pigeons received the value 1 and available sites were given 0 .

\subsubsection{Fixed-effects}

The independent variables were categorical variables measuring resources identified as influential to pigeon distribution. The habitat and resources measured (fixed-effects) were the proximity of pigeons groups $(<5 \mathrm{~m})$ to threats and other bird species, such as ducks, sparrows and seagulls (i.e., competitors); cafés, outdoor seating, people, rubbish bins, food waste, vegetation and water; and the habitat type they were found in. Resources within a 5 meter radius from the feral pigeon group were deemed to be selected. Identification of threats within five meters of the feral pigeons were recorded. Threats to feral pigeons included people and vehicles. Habitat type variables were recorded, such as public square or mall, building (modern or historic), bridge and wharf. The proximity of vegetation (tree, shrub, or grass) within 5 meters of the feral pigeon group was also 
recorded.

General information such as the weather conditions, including, temperature, wind speed were recorded for each transect. We did not conduct transects during poor visibility, such as when it was raining.

\subsubsection{Candidate models}

Hypotheses for feral pigeon groups' selection of resources and flock size were compared across seven different themes. Hypotheses were generated from the published literature and personal observation. The themes related to artificial food, people, roosting/nesting sites, vegetation (shrubs, trees, and grass), water (fresh water), competition (other bird species), and threats (vehicles and people) (Table 2.1).

The artificial food hypotheses focused on whether the presence of artificial food would increase the likelihood that pigeons would be present. Artificial food was divided into two models: (1) potential and/or opportunistic food, i.e. feeding; and (2) disposed food waste, i.e. scavenging. Sources of potential/opportunistic food included cafés, grassy areas where people sit to eat lunch, city squares and malls where people congregate, and outdoor seating where people potentially eat food. Sources of disposed food waste included discarded food and refuse bins.

In comparison to the models related to food, a hypothesis related to the presence of people (either positive or negative relationship) was modeled to further explain whether food or the people themselves primarily influence feral pigeon distribution. A model was created to deter- 
mine whether sources of shelter and roosting spots influence feral pigeon presence. Roosting spots and shelter included modern buildings, historic buildings, trees, wharves and bridges. Proximity to vegetation (trees, shrubs and grass) was included as a separate model to determine whether natural food sources, such as seeds, are likely to influence feral pigeon distribution. A model related to the proximity of fresh water to feral pigeons was used to determine whether it is likely to influence feral pigeon distribution. A model for competition of feral pigeons with other bird species was used to represent whether the presence of other birds is likely to influence the presence of feral pigeons. Other bird species in close proximity to a group of feral pigeons may be a source of competition and may displace pigeons from prime habitat and food sources.

\subsubsection{Statistical method}

We adopted an Information-Theoretic approach to testing hypotheses for feral pigeon group presence and size. An Information-Theoretic approach examines several competing hypotheses simultaneously to identify the best set of models (i.e. hypotheses) (Johnson and Omland, 2004), and is distinguished from traditional null hypotheses testing by quantitatively ranking and weighting each competing hypothesis or model versus null hypotheses testing where an arbitrary probability threshold is used to support a hypothesis (Burnham and Anderson, 2002). The advantage of the Information-Theoretic method for our approach is that it allows the comparison and influence from multiple, plausible models to be understood. 
Support for models representing hypotheses for site selection and flock size were then estimated using logistic regression (Keating and Cherry, 2004). Models for group size (congregation) were estimated by linear regression. The models were then compared using akaike information criterion (AIC). The best models were selected by balancing improvement of model fit by adding parameters with a reduction in the quality of the parameter estimates by having less data per parameter (Posada and Buckley, 2004). To aid model selection, each model was ranked and weighted to provide a quantitative measure of relative support for each competing hypothesis. $\triangle A I C<2$ was considered to have substantial support and $\triangle A I C>10$ was considered to have no support.

The data analysed excluded observations of pigeon groups that included missing values. Thirty-five observations out of 1068 total observations from the data set were removed because of missing values. Sites with missing values were removed completely from the analysis because a character of the site could not be observed. The small number of observations removed is unlikely to change outcomes of the compared models.

\subsection{Results}

\subsubsection{Site Selection}

A total of 1,068 observations were made to aid the site selection study. The model describing opportunities for food from people was the only model to receive substantial support and all Akaike weights ( $\triangle \mathrm{AIC} \leq 2$, 
$W=0.999$ ) (Table 2.1). Proximity to places where people congregate to eat, such as outdoor seating and cafés, was the primary factor that influenced feral pigeon site selection (Figure 2.1). Threats such as traffic did not change the likelihood that pigeons would be present. The availability of roosting/nesting sites, other birds as potential competition, rubbish bins, availability of water, vegetation/grass, and proximity of people did not explain feral pigeon habitat selection $(\triangle \mathrm{AIC} \geq 10, W<0.0001)$. In this study, the availability of nesting or roosting sites had less than 1\% Akaike weight, which indicates that groups of pigeons are unlikely to select resources solely based on the availability of roosting or nesting sites.

\subsubsection{Flock size}

Flock sizes ranged from a single pigeon to over 50 pigeons. The models for tertiary vegetation and disposed food performed the best $(W=0.324$ and $W=0.297$, respectively) (Table 2.2). These results indicate that larger groups of feral pigeons may be more likely to select areas with food waste and tertiary vegetation, such as trees. However, a number of the other models provided explanatory power to larger group selection of resources (Table 2.2). The $R^{2}$ values for each of the models were low so each has poor predictive power. Therefore, a number of variables are driving flock size of feral pigeons. 


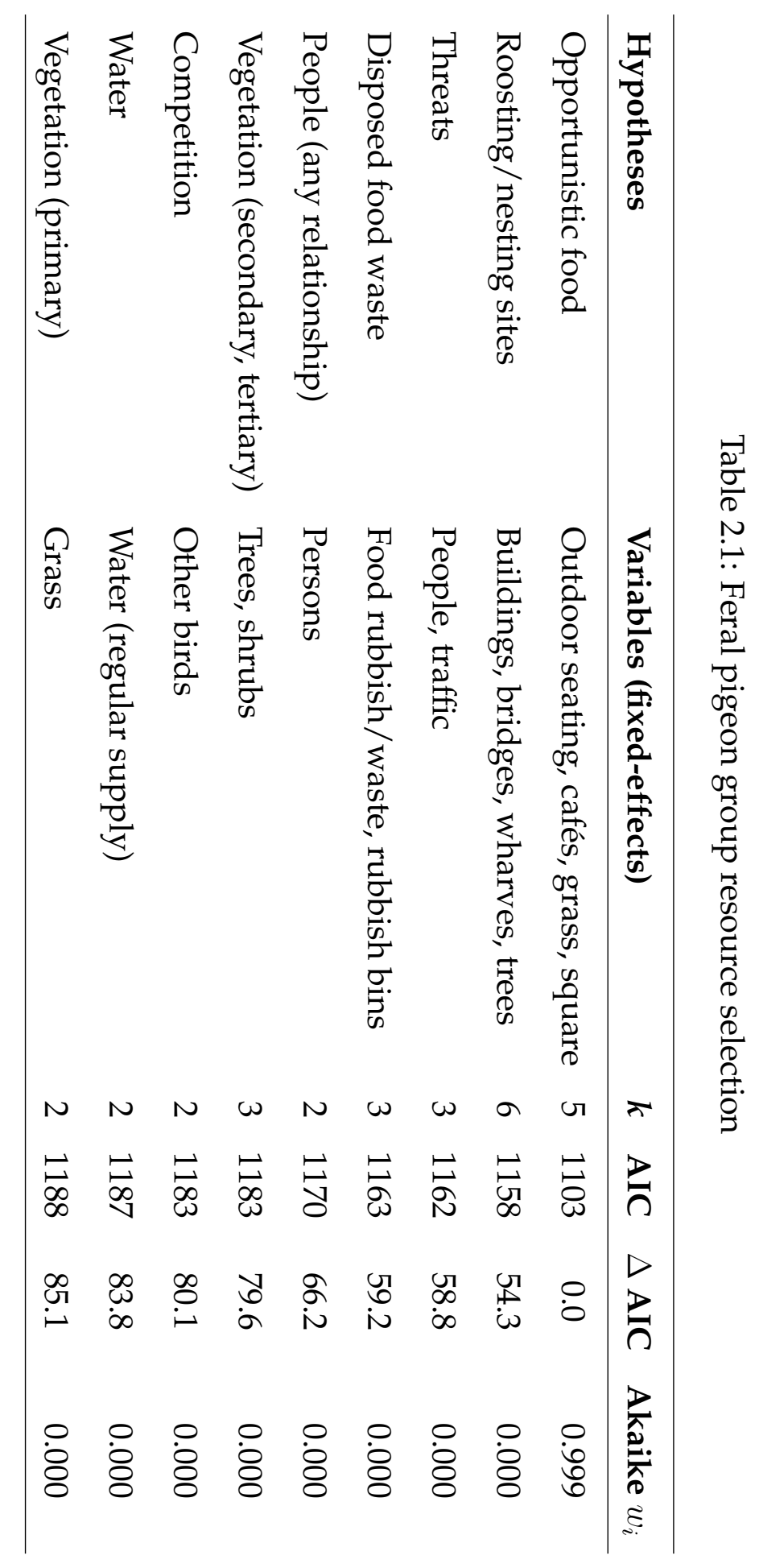




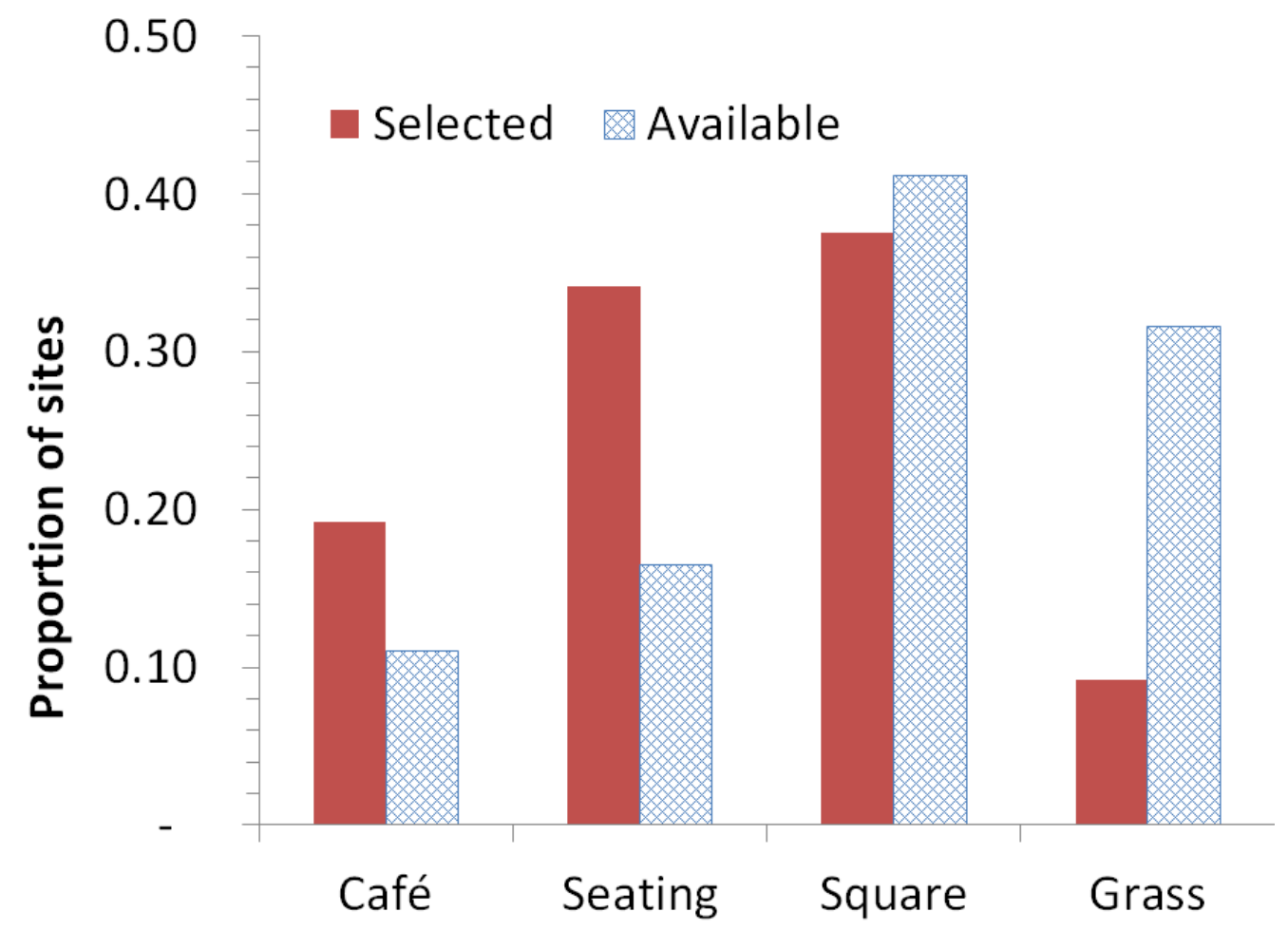

Figure 2.1: The proportion of sites that were selected and available in central Wellington City for cafés, outdoor seating, squares and grassed areas ( $n=193)$. The sites are from the best performing model ( $\Delta$ AIC $\leq 2$, $W=0.999)$, which suggests that areas where people congregate to eat is the primary factor influencing feral pigeon site selection. The survey consisted of eight line transects through central Wellington and was completed in summer (February - March) and winter (July - August) over a 2-3 week period. 


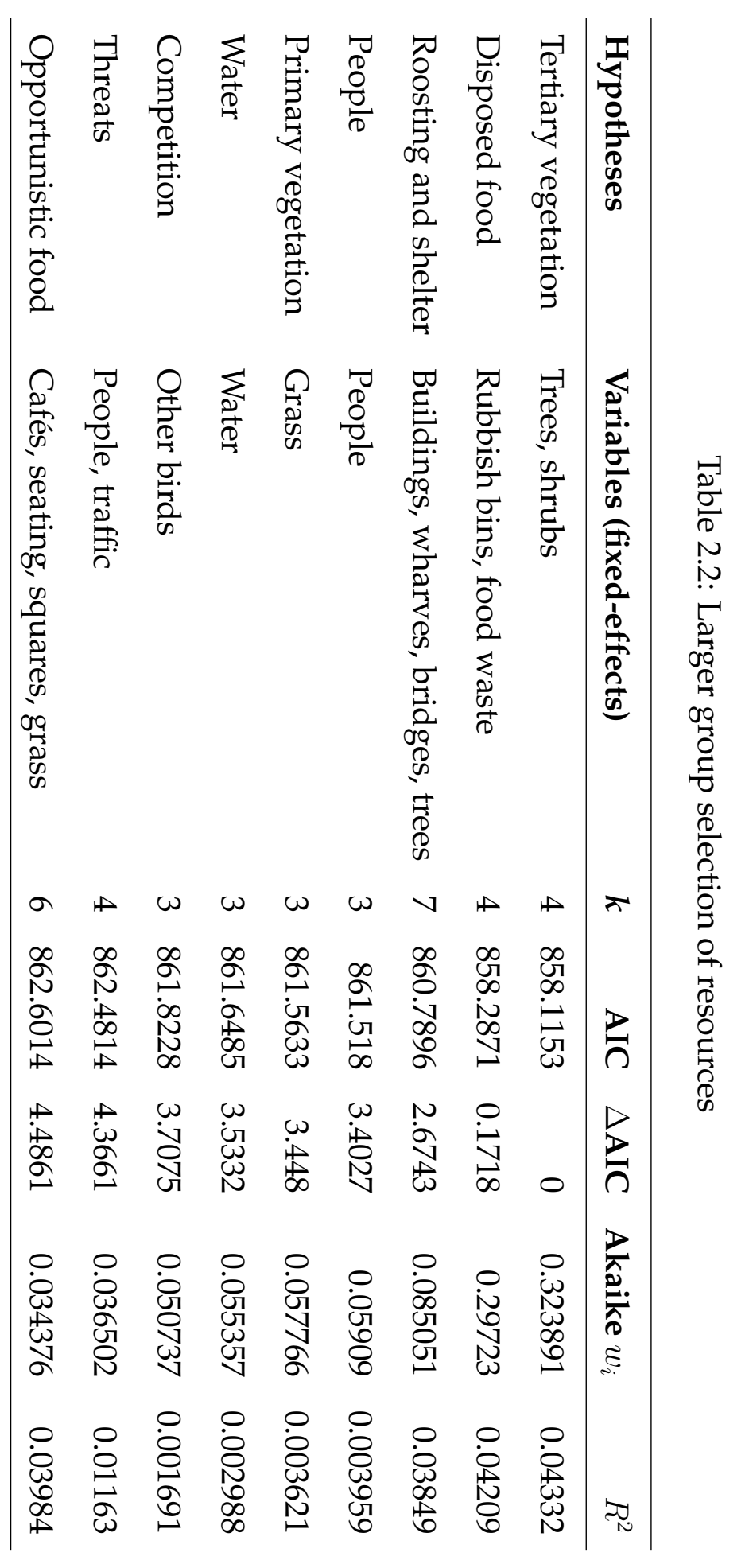




\subsection{Discussion}

This study explains how feral pigeons use urban environments and the resources key to their distribution and congregation. The results show that feral pigeon site selection may be primarily influenced by people and where they choose to eat. Feral pigeon resource selection, therefore, appears to be mostly determined by sites where food is provided by people, i.e. through feeding. These results suggest that feral pigeon populations may be largely dependent on the availability of anthropogenic foods and that a reduction in food provided by people may limit feral pigeon populations.

Feral pigeon populations generally increase towards the centre of cities (Johnston and Janiga, 1995) and with increases in the density of people (Buijs and Wijnen, 2001; Jokimäki and Suhonen, 1998), but it is unknown why this variation occurs. The main factors invoked to explain the relationship are greater food-resource accessibility and higher availability of roosting-breeding sites (Johnston and Janiga, 1995; Sacchi et al., 2002). These factors suggest a link between pigeons and people, but the cause of the link is unclear.

Our results suggest feral pigeons are attracted to people because of anthropogenic food. It is not just people that attract pigeons, but people's food. Pigeons are more likely to be in areas where people feed them or have the ability to steal food from people, i.e. from café tables. The likelihood of the presence of groups of feral pigeons in Wellington City is best explained by areas where people congregate to eat food. This means that 
feral pigeons are less likely to choose habitat where people just congregate but do not eat, i.e, sidewalks.

The results of this study align with a study done in Barcelona, Spain (Sol et al., 1998). The Spanish study found that pigeons were largely dependent upon two types of food sources: (1) food provided by people; and (2) waste food produced by human activity. Sol, et al, distinguished the two types of food according to how they are distributed in space and time. Food provided by people was given in large quantities but available only for a short time in limited areas. Most of this food was provided daily at the same place and time by the same people. Waste food was available for a long time and widely dispersed but at very low concentrations. As a result, the two food types require different foraging techniques: food provided by people only requires pecking at food items, and waste food requires pigeons to search for food items (Sol et al., 1998).

My study is consistent with the findings of Sol et al that found that feral pigeons prefer food provided by people rather than waste food. Our results confirmed that pigeons prefer to be in areas where people are likely to be eating than near rubbish bins or other areas with waste food. As a result, feral pigeons in Wellington City are less likely to have to spend their time searching for small amounts of waste food. Feral pigeons are more likely to select areas where people consistently feed pigeons, so even though they still have to search for people, people are usually in predictable areas with food provided at regular times each day.

This study is in contrast to other studies that suggest a strong link be- 
tween feral pigeons and historic buildings (Sacchi et al., 2002). Urban centres are resource rich for roosting and nesting sites, such as buildings and bridges. Our results indicate that feral pigeons were not resource limited for roosting sites. Roosting sites are plentiful in urban Wellington City and are not the primary reason that feral pigeons select habitat.

Feral pigeon distribution does not seem to be affected by threats. Threats, such as traffic and people, do not appear to significantly influence feral pigeon habitat selection. In the areas where people congregate to eat, there is usually not vehicular traffic. Fast moving human traffic that creates the potential to trample pigeons is also much more limited in those areas compared to footpaths, e.g., squares and parks are not usually main thoroughfares.

Similarly, feral pigeons do not seem to be affected by competition from other birds. Other birds, such as seagulls and sparrows, do not appear to affect whether pigeons are likely to select or avoid a site. This may be because there are limited numbers of competitors that use the same sites as feral pigeons. Alternatively, feral pigeons may be much more successful at sourcing food from people than other birds and, therefore, do not have to change their behaviour at sites with other birds.

Freshwater is important for feral pigeons, particularly during breeding season as it provides optimal food for young (Johnston and Janiga, 1995). A pigeon needs 30-50 grams of water per day and they usually drink twice a day from rain puddles, ponds, streams, or fountains (Dorzhiev, 1991). A small portion of their water requirement comes from their food such as 
seeds. Pigeons are unique among bird species for their ability to drink without lifting their heads. The pigeons' beak is similar to a straw that is able to suck water from tiny sources, such as puddles. The presence of permanent freshwater sources (e.g., fountains) was found unlikely to influence feral pigeon habitat selection. Feral pigeons do not have to rely on permanent water features because of their ability to drink from puddles and small patches of water without having to raise their heads to drink like other bird species. This allows feral pigeons much more freedom of movement because they are not limited to areas with plentiful water sources. Whereas other birds may not be able to exist in areas where water sources are shallow or temporary, which may depend upon frequent rainfall. In climates that receive rain throughout the year, like Wellington City, water features are unlikely to significantly influence feral pigeon habitat selection.

The vegetation hypothesis received no support, with feral pigeons unlikely to select habitat on the basis of shrubs, trees or grass.

This study also highlights an important distinction that was apparent from using Information-Theoretic analysis versus null hypothesis testing. The data showed significance of a number of resource factors that were not indicative of best fit unless combined into a single model. From hypothesis testing, you might conclude that the significance of people is what is determining feral pigeon resource selection. However, when you separate people from areas where people eat (e.g., outdoor seating), the model performs much better. Therefore, future studies should take a cautious 
approach when using null hypothesis testing. 


\subsubsection{Flock size}

The flock size of feral pigeons needs special attention. Flock size is associated with the amount of damage that feral pigeons can cause to buildings, aggregation of droppings, and disturbance to people. Flock feeding in birds has several advantages such as protection against predators, increased feeding rate or feeding time, and increased access to new food sources through local enhancement (Lefebvre, 1983). Urban pigeons usually feed in flocks (Murton et al., 1972b). The largest flocks of feral pigeons have been shown to be correlated with historic buildings (Sacchi et al., 2002), which frequently exist in urban centres such as Wellington City.

In Wellington City, site selection by groups of feral pigeons is mostly influenced by areas where people congregate to eat. However, after a habitat is selected, feral pigeon flock size is influenced by a number of habitat characteristics (in contrast to habitat selection where a single variable provides the most explanation). Group size is determined by a combination of variables, with tertiary vegetation and food waste receiving the strongest weighting. Tertiary vegetation (shrubs and trees) may be ideal for larger flocks of feral pigeons for cover and roosting. Indeed, at some sites in Wellington City, large numbers of feral pigeons congregate in trees (based on personal observation during 2009-2010). Food waste indicates that people may eat in the area and therefore attracts larger numbers of pigeons. Permanent water features may also support larger flocks of feral pigeons as there is no need to seek out water multiple times a day. Therefore, sites with a number of resources may attract larger flocks of feral pigeons. 


\subsection{Management implications}

Many cities around the world have problems with feral pigeons (Krebs, 1974). Wildlife management professionals in urban centres have attempted various methods of feral pigeon control. Methods used include direct control such as shooting and euthanasia (Murton et al., 1972a), removal of eggs from nesting areas (Haag-Wackernagel, 1995), and provision of birth control (Dobeic, 2003). Each of these methods have indicated limited success. The provision of birth control through hormonally treated maize resulted in a 24.3 per cent decrease of feral pigeons across three years in Ljubljana, Slovenia (Dobeic, 2003). Using birth control is a very resource intensive method of control and required daily feeding of pigeons during mating seasons. As a result, this method may not be achievable for most cities. Other methods, such as direct control have proven much less successful than the expensive birth control option. Studies have found that new immigrants arrive to take the place of the removed birds (Murton et al., 1972a; Sol and Senar, 1995). Whenever control operations were relaxed, feral pigeons numbers increased rapidly due to year-round breeding and influxes of pigeons from other areas. Culling is also resource intensive because of the need for ongoing removal of feral pigeons at regular intervals. Studies have shown that management costs can be as high as nine dollars a pigeon per year (Haag-Wackernagel, 1995). Central city feral pigeon population may be as dense as 6.8 pigeons per hectare in Wellington City, thus making culling operations financially prohibitive (Ryan, 2011).

The method used in Basel, Switzerland involved setting up a loft for 
feral pigeons (Haag-Wackernagel, 1995). Wildlife managers regularly remove eggs from pigeons' nests and replace them with plastic eggs. This method is also resource intensive and costly and can meet with limited success in areas where there is not a shortage of nesting spots. The feral pigeon loft in Melbourne, Australia has met with limited success despite feeding feral pigeons at the loft to attract them (TheAge, 2007). Because these results indicate that feral pigeons in Wellington City are not limited in the availability of roosting and nesting spots, this method may have limited success in Wellington City. However, this method meets animal welfare concerns and is popular with the general public in Basel, Switzerland. It allows for the public to enjoy having a limited number of healthy feral pigeons in their town.

Urban authorities that have problems with large groups of feral pigeons can concentrate efforts in areas where people congregate to eat that have food waste (i.e., rubbish bins) and roosting sites (i.e., buildings with ledges or trees). Therefore, urban authorities should focus on areas with the highest number of these resource types to manage large flocks of feral pigeons.

This study suggests that modification of people's habits to feed pigeons could result in lower numbers of pigeons in Wellington City. Urban wildlife management could focus on changing people's feeding behaviour rather than culling, which meets public opposition and limited effectiveness. By modifying the amount of food that people provide to feral pigeons, pigeon populations may decrease. Populations may decrease be- 
cause feral pigeons are relying on anthropogenic foods and any decrease in food may limit their ability to breed year-round or lower survival rates. The results indicate that urban authorities should focus their efforts in areas where people are likely to eat rather than limiting availability of roosting or nesting areas through spikes or nets. Some urban centres have focused on providing alternative nesting areas, i.e., pigeon lofts, which may only work in areas where there is limited supply of nesting areas. Feral pigeons tend not be too particular about their nesting spots, with many using gaps and openings in buildings, and the results reflect that feral pigeons in Wellington City are not limited by availability of roosting options. By focusing on areas where people are likely to congregate to eat, it allows for city authorities to put more effort into educating the public in those areas, which may include signs instructing people not to feed pigeons, and increasing the number of rubbish bins (that are inaccessible to pigeons) for people to dispose of food. Public education and efforts to contain food waste may be much more cost effective and durable than ongoing removal of feral pigeons or maintenance of feral pigeon lofts. Addressing pigeon problems by changing people's feeding behaviour meets animal welfare concerns for wildlife management. It is also a much more politically viable option than culling, which often gathers much public opposition. If people keep their food inaccessible and limit any direct feeding to feral pigeons, it may provide a management solution for feral pigeon problems in cities around the world. 


\section{Chapter 3}

\section{Anthropogenic food availability and feral pigeon condition and activity}

\subsection{Introduction}

Whether or not wildlife are food limited in an urban environment is a key question for urban ecologists. Food provided by people has a major effect on density and distribution of birds in urban areas (see Chapter 2 herein) (Lancaster and Rees, 1979). Urban environments have high amounts of anthropogenic foods (Jokimäki and Suhonen, 1998; Anderies et al., 2007) and limited predation (Shochat, 2004). Therefore, some bird populations in urban habitats reach high densities (Valcarcel and Fernández-Juricic, 2009), especially introduced and commensal species. Species that receive supple- 
mentary food usually have smaller home ranges and heavier body weights (Boutin, 1990). Even moderate amounts of additional foods can greatly increase reproductive success (Boutin, 1990). In a supplementary food study on song sparrows, supplementary food advanced laying date, increased clutch size, increased the number of breeding attempts, increased nestling weight, and increased the number of independent young produced, reduced brood parasitism, and reduced the interval between successful nesting attempts (Arcese and Smith, 1988). Also, juveniles receiving supplemental food have better survival and so population recruitment rates are higher (Smith et al., 1980).

One of the most successful colonisers of urban environments is the feral pigeon (Columba livia) (Haag-Wackernagel et al., 2006). Compared to the domestic variety, feral pigeons fly faster, have a higher annual reproductive potential, and greater genetic variability (Adams et al., 2006). Feral pigeons have adapted their original diets of grain to include anthropogenic sources of food. Urban feral pigeons may depend on the public for food sources or steal food from grain storage areas, e.g. bakeries (Murton et al., 1972b). Wellington is an ideal city to study feral pigeons because, unlike in other cities, Wellington is not surrounded by intensive agriculture, such as mixed cropping. Consequently, pigeons may be unlikely to leave the city to feed in agricultural fields for the day and return at night to nest. And, in areas outside of Europe such as Canada, India, and New Zealand, feral pigeons do not readily adjust to eating seeds of native plants (Johnston and Janiga, 1995). Therefore, feral pigeons in Wellington City may rely solely 
on anthropogenic food.

Understanding how food shortages affect urban bird species is important for urban wildlife management because variations in food supply is the primary factor effecting population size in birds (Arcese and Smith, 1988). This study investigated whether the quantity of feeding by people at central Wellington sites affected feral pigeon (1) condition and (2) activity; with the general hypothesis that food supply may limit Wellington City's pigeon population. I predicted that feral pigeon condition (body mass divided by tarsus length), plumage condition, and keel condition would be higher at sites with higher levels of public feeding; birds at higher-fed sites would spend more time resting (e.g., roosting or sitting) than birds at lower-fed sites, who would have to spend more time searching for food. My food limitation hypotheses is an alternative to the ideal free distribution theory. Under the ideal free distribution theory (Sol and Senar, 1995), body condition across sites may be similar because of how birds distribute themselves to receive an optimal amount of food. Under the ideal free distribution theory, I would expect no difference in condition because birds will redistribute themselves among sites to get the optimal amount of food. 


\subsection{Methods}

\subsubsection{Study site and population}

This study was carried out in central Wellington City. Wellington City is New Zealand's third most populous city with 195,000 people living in the city. The City is located on the edge of a harbour and is surrounded by steep hills and has a mild temperate, oceanic climate. It is well-known for its strong winds (predominantly northerly) and hilly topography. The climate is sunnier than other cities with feral pigeons and has over 2,000 hours of sunshine a year (compared with 1,500 for London). The coldest month is July with an average temperature of 8.7 degrees Celsius and the warmest month is February with an average temperature of 17 degrees Celsius. The average rainfall in the Wellington region is $1,270 \mathrm{~mm}$ and is distributed throughout the year with slightly more rain in winter than summer. Feral pigeons are resident year-round in Wellington City and are capable of breeding year-round in New Zealand (Dilks, 1975).

\subsubsection{Experimental design}

I conducted an observation-based experiment utilising fortuitous variation in anthropogenic food availability. Observations were done across a number of Wellington City parks to test the hypothesis that food supply may limit Wellington City's feral pigeon population. The study was conducted during winter - approximately late-June through August 2010. Winter was selected as the best season to carry out the study because it 
is the period of the year when food resources of all types are expected to be most limited. There is less natural food in winter because plants have limited growth and are not producing any seeds. There may also be less anthropogenic food available in winter because people are not eating outside as often.

Five sites across the central city were selected to display a range of anthropogenic food levels. Each site had similar characteristics such as outdoor seating, grassy areas for people to sit, and potential roosting sites (e.g. trees or building ledges) for feral pigeons. Each site was visited multiple times a week at various times of day from June through October. During each site visit, the presence or absence of anthropogenic food was recorded and quantified. The quantity of anthropogenic food at each site was recorded on a scale from one to five, with one being a small amount of food. A small amount of food was noted as crumbs of bread or remnants of seed. A large amount of food was multiple loaves of bread or cups of grain. Sites were then categorised as low, medium or high fed according to the average amount of food seen during June-October.

A total of 48 feral pigeons were caught using mist nets and hand nets (41 adults and 7 juveniles) and were banded using metal and colour bands at each site. Each bird was given two bands on each foot. Feral pigeons were weighed and measured. And, keel and feather condition were rated. Measurements taken included the length of the tarsus, length of wing, length of tail, length of bill, and width of bill. Body condition was estimated by overall body mass, keel condition and feather condition. Feather 
condition was rated by the presence or absence of tears and stains.

Animal weight incorporates variation in both body size and condition, and, therefore, it is not a good measure of size or condition (Sutherland et al., 2004). Body mass corrected for size gives a better indication of the bird's condition (Owen and Cook, 1977). We corrected for differences in body size among birds by dividing body mass by tarsus length (Brown, 1996).

The wing was measured using the distance from the carpal joint to the tip of the longest primary feather on the closed wing. A stopped stainlesssteel ruler with the wing held in its natural closed position, or as near to it as possible, was used to measure the wing. Measurements were recorded to the nearest $0.5 \mathrm{~mm}$. The birds were released immediately after measurements were taken and the bands attached.

\subsubsection{Statistical method}

A non-parametric Kruskal-Wallis one-way analysis of variance (ANOVA) test was used to study the difference in feral pigeon condition and activity across sites. The test used a single factor with three levels: one for each of the sites (low, medium and high fed).

\subsection{Results}

The quantity of food at each of the five sites was ranked as low, medium and high. Two pairs of sites had similar quantities of food and were cate- 
gorised similarly.

Feral pigeon condition of both adults and juveniles was not significantly different between sites $(n=48)$ : body condition (body mass/tarsus length), Kruskal-Wallis = 2.06, $\mathrm{p}=0.36$ (Figure 3.1); keel condition, KruskalWallis $=0.7283, \mathrm{p}=0.6948$ (Figure 3.2); feather condition, Kruskal-Wallis = 2.7943, $\mathrm{p}=0.2473$ (Figure 3.3).

The activity of feral pigeons (resting and foraging) across sites was not significantly different: $\mathrm{n}=285$, Kruskal-Wallis $=1.6875, \mathrm{p}=0.2078$, (Figure 3.4). The results did not change when juveniles were excluded for any of the analyses. 


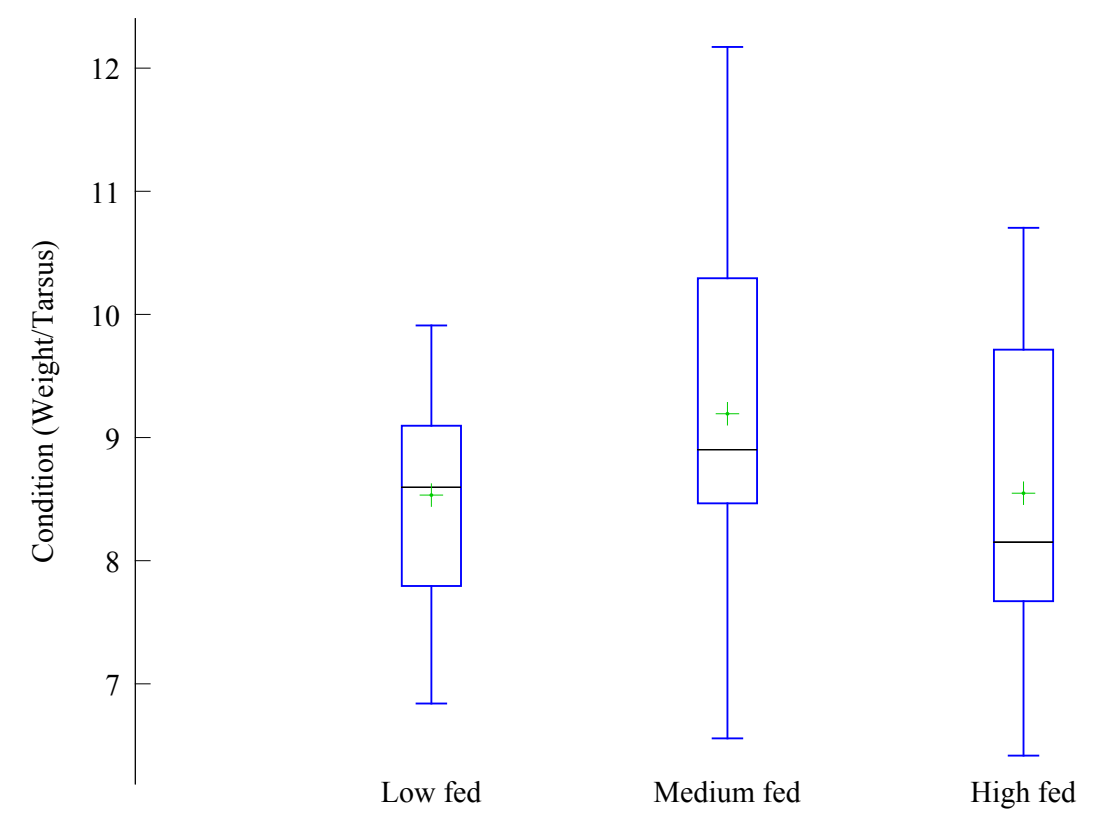

Figure 3.1: Distribution of results of feral pigeon condition (body weight/tarsus) across three sites in Wellington: low $(n=12)$, medium ( $n=18)$ and high $(n=18)$ fed. Each site was categorised as low, medium or high fed by observation of the amount of anthropogenic food seen between June and October 2010. Birds were banded $(n=48)$ at each site using mist and hand nets in winter 2010. Body weight was measured using a scale. The box and whisker plots indicate the average by the + symbol and the median by the line in the middle of the box. The boxes show the 25th quartile and the 75th quartile with extensions showing the range of values. 

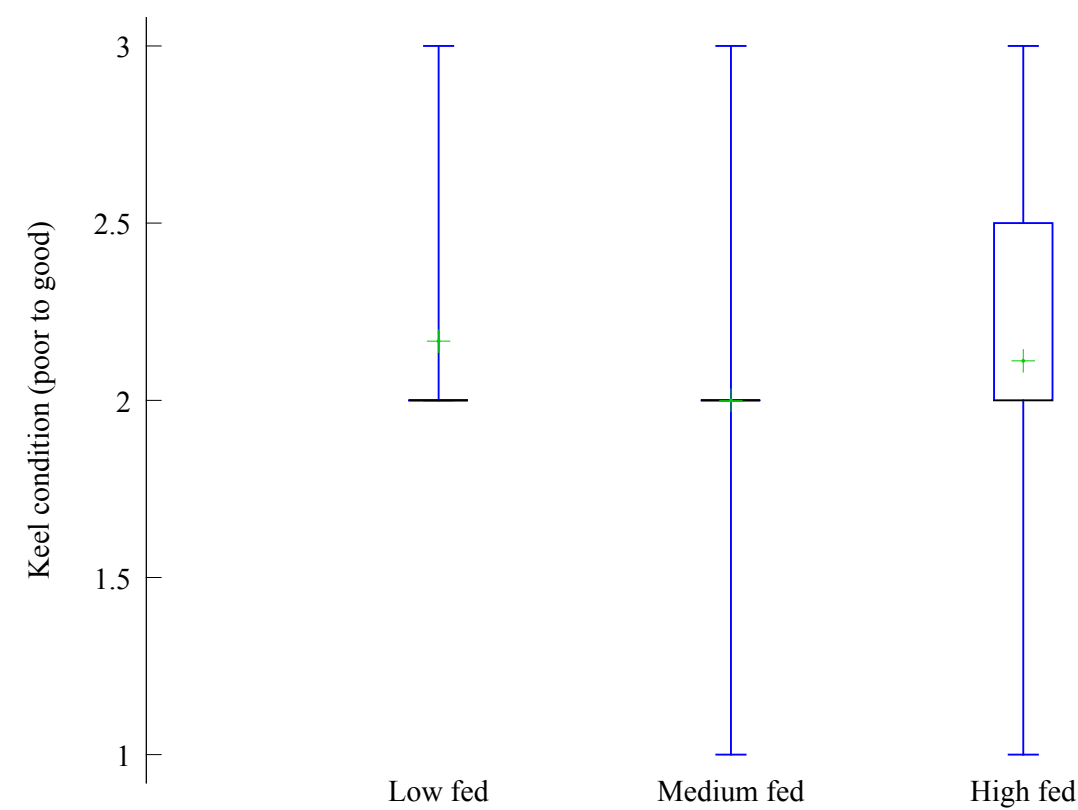

Figure 3.2: Distribution of results of adult feral pigeon keel condition across three sites in Wellington: low $(n=12)$, medium $(n=18)$ and high $(n=18)$ fed. Each site was categorised as low, medium or high fed by observation of the amount of anthropogenic food seen between June and October 2010. Birds were banded at each site using mist and hand nets in winter 2010. Keel condition was estimated by the amount of fat felt with two fingers. The box and whisker plots indicate the average by the + symbol and the median by the line in the middle of the box. The boxes show the 25th quartile and the 75th quartile with extensions showing the range of values. 

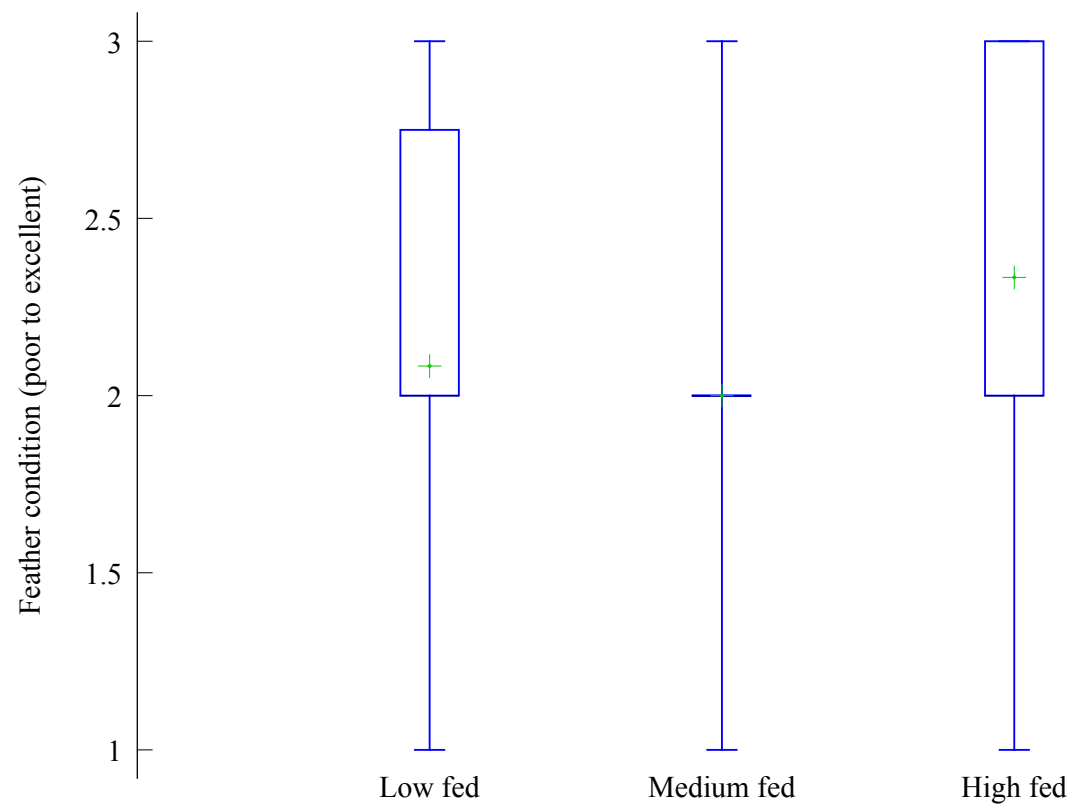

Figure 3.3: Distribution of results of feral pigeon feather condition across three sites in Wellington: low $(n=12)$, medium $(n=18)$ and high $(n=18)$ fed. Each site was categorised as low, medium or high fed by observation of the amount of anthropogenic food seen between June and October 2010.Birds were banded and measured at each site using mist and hand nets in winter 2010. The box and whisker plots indicates the average by the + symbol. The boxes show the 25 th quartile and the 75 th quartile with extensions showing the range of values. 


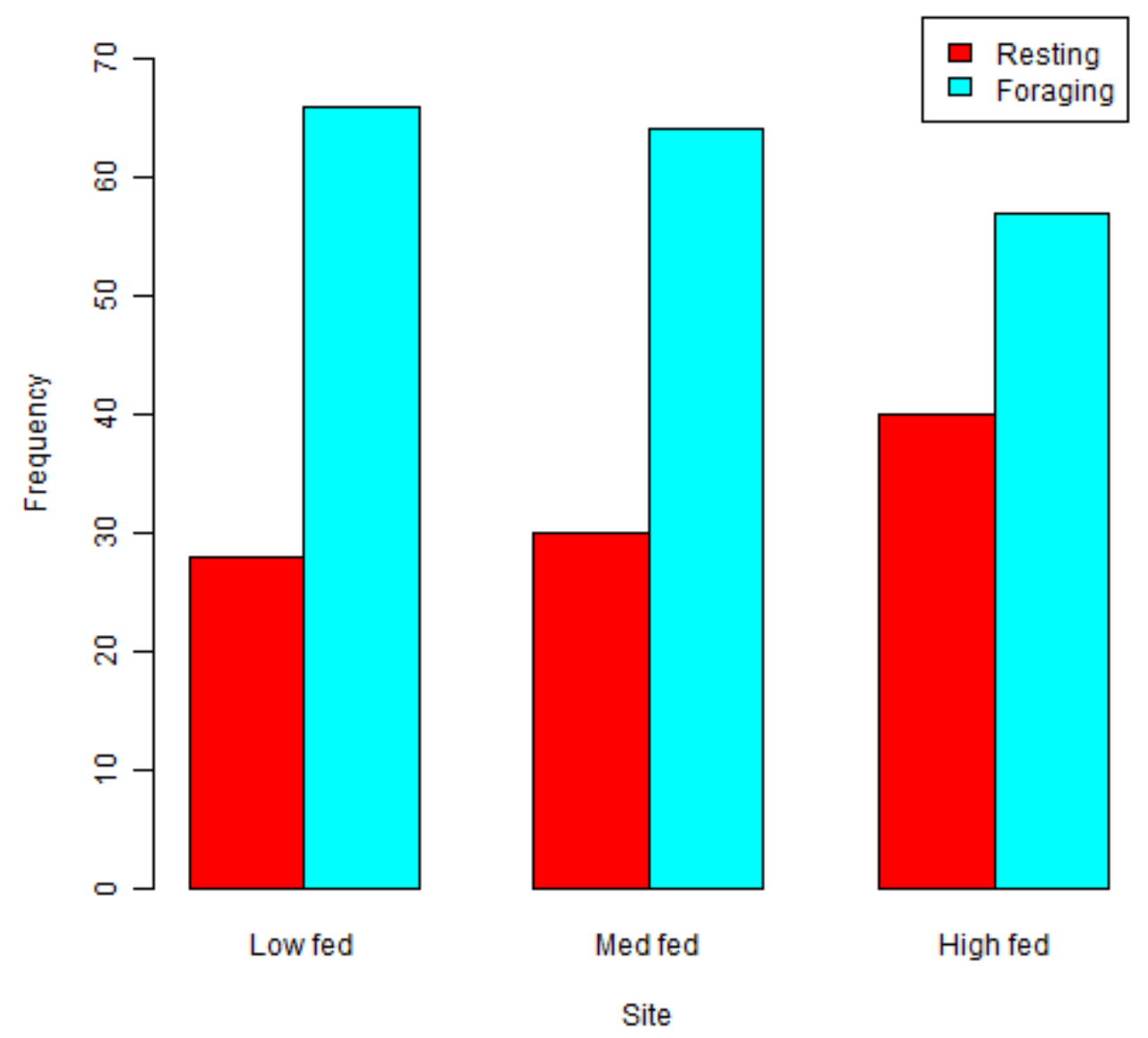

Figure 3.4: Frequency of feral pigeon activity across three sites in Wellington: low, medium and high fed. The level of feeding at each site was quantified by observation into low, medium and high fed. Birds were banded and measured at each site using mist and hand nets in winter $2010(n=48)$. Activities (resting or foraging) of feral pigeons throughout Wellington were recorded during winter and spring $2010(\mathrm{n}=285)$. 


\subsection{Discussion}

It is not well understood whether urban environments are resource limited for feral pigeons. Anthropogenic foods provided by people may determine the population size and growth of feral pigeons. Limited food resources means that feral pigeons will invest less time in breeding (e.g., because they must search longer for food) (Rose et al., 2006). Food can be considered limited if it prevents a population from increasing (Newton, 1980). The results of this study will provide information to wildlife managers on how anthropogenic foods influence feral pigeon populations. Limiting feral pigeons' food sources may be a more effective way to control population size than culling and it also meets animal welfare concerns (Haag-Wackernagel, 1995).

The study examined whether birds located at higher fed sites would be in better condition and spend more time resting than birds at lower fed sites. However, the study provides little evidence for differences in feral pigeon condition or activity levels among sites. Feather condition and body condition were similar across all of the sites. There was no significant difference between sites with low amounts to high amounts of anthropogenic food.

Feral pigeons at higher fed sites appear to spend more time resting, e.g., roosting, than those at lower fed sites (Figure 3.4). However, activity levels between sites were not significantly different. I hypothesised that birds at sites with high amounts of anthropogenic food would spend more time engaging in resting activities, such as roosting. However, the results 
show that birds at sites with low amounts of anthropogenic food may not spend any additional time searching for food than birds at the sites with high amounts of anthropogenic foods.

The results indicate that feral pigeons may not be food limited in central Wellington. Anthropogenic food appears to be plentiful. Food quantity at sites was based on what was left behind by the pigeons. Indeed, I observed uneaten food at all sites, which might indicate a satiated population, rather than unexploited food. The consistency of feral pigeon condition and activity levels across the sites suggests that food is either sufficient to satiate the population at each site (even those with low amounts of artificial food) or feral pigeons are travelling to other sites to feed.

Pigeons may be travelling to nearby sites to supplement the food they are receiving at lower fed sites. Travelling between sites to take advantage of the sites that offer the most amount of food is consistent with the ideal free distribution theory. The ideal free distribution theory supports the hypothesis that pigeons will distribute themselves equally per unit resource among sites to maximise individual food consumption (Sol and Senar, 1995). Therefore, feral pigeons may sample several sites and use cues of competitor density, feeding payoff and relative payoff (Lefebvre, 1983).

That a portion of the population travel between sites to feed is supported by the literature. Feral pigeons' social structure does not prevent pigeons from leaving their particular site or group to feed at other sites. Other studies have shown that feral pigeons have highly individual for- 
aging strategies and may join different feeding flocks (Rose et al., 2006). Feral pigeons may visit several feeding sites (Palameta and Lefebvre, 1985) and can adapt to a reduction in food by searching outside of normal or predictable food source sites (Murton et al., 1972a). Nevertheless, other observations indicate a large portion of the population to be site loyal and this is the case in Wellington too (Ryan, 2011). Alternatively, it might just indicate that pigeons do not have to travel widely to satisfy their food requirements.

The results indicate that urban wildlife management authorities should not focus on particular urban parks for feral pigeon control. A consistent approach across sites in Wellington is necessary because feral pigeons do not appear to be food limited at particular sites. Control of anthropogenic sources of food will need to be broad-based. If anthropogenic food is the main source of food, and that appears to be the case (see Chapter 2 herein), then reducing public feeding behaviour becomes a viable strategy for reducing pigeon populations so long as landscape-scale limitation is imposed. A landscape-scale strategy is necessary because control of food at one site, may only cause feral pigeons to move to other sites for food and may not limit population size.

Feeding by people is so extreme that food is left uneaten at sites in Wellington City. As a result, the pigeon population is likely to be satiated. The implications of a satiated population, not just food limited, include rapid reproduction, large flocks, high densities, health risks of carcasses and feces, greater damage to infrastructure, and increased human conflict. 
Management to reduce public feeding is advisable. See Chapter 2 herein for a discussion on feral pigeon management options. 
52 CHAPTER 3. ANTHROPOGENIC FOOD AND URBAN WILDLIFE 


\section{Chapter 4}

\section{Attitudes of businesses towards}

\section{urban wildlife}

\subsection{Introduction}

In New Zealand, almost three-quarters of people live in the main urban centres (Statistics, 2006) and there is an increasing urban population worldwide. The shift to urban populations requires a fundamental change in urban wildlife management and decision making that involves the public (Patterson et al., 2003). Urban perspectives on wildlife are highly individualised with differing emotional and symbolic values (compared to rural or agricultural societies who often share the same meaning and values towards wildlife), which may increase the likelihood of social conflict over wildlife management (Patterson et al., 2003).

With the majority of people living in cities, conservation initiatives may 
largely depend on people who live in cities with reduced experience of wildlife and wildlife habitat. The success of conservation initiatives may be linked to people's interactions with urban wildlife (Dunn et al., 2006). Interactions with wildlife can influence people's attitudes and willingness to contribute to protecting endangered species (Ericsson and Heberlein, 2003). Attitudes have been shown to be a powerful predictor of human behaviour (Balram and Dragievi, 2005). The term attitude can be defined as tendencies to evaluate a particular action or outcome with some degree of favour or disfavour (Eagly and Chaiken, 1993). If urban residents have negative attitudes towards urban wildlife, such as feral pigeons, it may influence their willingness to participate in conservation initiatives. Negative attitudes towards urban wildlife may cross over to other species of wildlife, including native and endangered species.

One of the most successful colonisers of urban environments is the feral pigeon (Columba livia) (Haag-Wackernagel et al., 2006). Feral pigeons have adapted their original diets of grain to include anthropogenic sources of food. Urban feral pigeons may depend on the public for food sources or steal food from grain storage areas, i.e., bakeries (Murton et al., 1972b). Feral pigeons have also readily adapted to urban infrastructure in place of traditional cliff-dwelling locations to use as roosting and nesting spots.

The general public's attitudes towards feral pigeons are important because the general public will often be the instigators of pigeon control (Jerolmack, 2008). Those most likely to be affected by pigeons in urban centres are local businesses, particularly food establishments, such as restau- 
rants, cafés and bakeries. Food establishments play a special role with urban wildlife management issues because they are more likely to have interactions with urban wildlife. For this study, food establishments may be more likely to have a high level of interaction with feral pigeons compared with other members of the community. Many of those interactions may be negative, e.g., conflict with wildlife attempting to obtain food from their business. Food establishments are likely to bear the cost of cleaning up after pigeons, deterring pigeons from their grounds, and limiting pigeons' access to food.

Food establishments are key members of the city business community. Business leaders have influential positions in the community and the financial means to contribute to conservation initiatives. Research has shown that individuals' attitudes about an issue influence behavioural intentions toward that issue (Stern et al., 1995). Wildlife management research has also found that attitudes and norms can influence managers' attention to environmental issues (Henriques and Sadorsky, 1999). Individual business leaders' attitudes on the environment are particularly important for changing organisations, such as food establishments (Schelly et al., 2011). The environmental attitudes of business leaders (and employees) influence the success of sustainable practices. Therefore, understanding what shapes people's attitudes, especially those in business, is important.

People's attitudes are formed through a variety of dimensions, such as knowledge, direct experience, values, beliefs and affiliations (Cordano 
et al., 2004) as well as place attachment (Vorkinn and Riese, 2001). Some research has demonstrated that behavior is more accurately predicted from attitudes formed via direct, behavioral interaction with the attitude object than from attitudes developed via indirect, nonbehavioural experience (Fazio and Zanna, 1981). Therefore, people's interactions with pigeons may be more likely to influence their attitudes than other indirect experiences.

This chapter seeks to understand how owners and managers of food establishments' interactions with feral pigeons influence their attitudes towards feral pigeons. Particular attention will given to whether direct behavioural experience influences attitudes towards feral pigeons. The paper will also identify solutions for city wildlife management authorities to improve business attitudes towards feral pigeons.

\subsection{Methods}

\subsubsection{Study area and population}

Wellington City is New Zealand's third most populous city with 195,000 people living in the City. The City's population is young with 55.9 percent aged between 18-49 years old. It has a higher proportion of Europeans (76.8 percent) than New Zealand as a whole. Wellington City residents are highly educated with over 33 percent holding a university degree. Incomes in Wellington are above the national average, with over 40 percent of households having an annual income over $\$ 89,000$. 
Wellington City sits on the edge of a harbour and is surrounded by steep hills and has a mild temperate, oceanic climate. It is well-known for its strong winds (predominantly northerly) and hilly topography. There are a high number of vegetated (green) spaces surrounding the city and there are many urban parks. The City has a high number of restaurants and cafés, with an estimate of more cafés per capita than New York City (Council, 2010). And, the City has a number of initiatives to support sustainable businesses (Council, 2010).

\subsubsection{Sampling}

Food establishments were randomly selected from the Yellow Pages telephone directory (YellowPages, 2010). Google Maps (Google, 2010) for Wellington were also used to identify food establishments in Wellington. Participants were selected based on the classification that food is sold on the premises and included takeaway food establishments, cafés, and restaurants. Only establishments that are open during the day (at least from noon onwards) were included. Location also limited participants by restricting the survey area within Wellington's main suburbs including, Wellington central, Te Aro, Thorndon, Pipitea, Aro Valley, Kelburn, Newtown, Miramar, Strathmore, Lyall Bay, Brooklyn, Hataitai, Island Bay, Wadestown, Karori, Khandallah, Ngaio, and Petone. The questionnaire was targeted at those who have a financial interest in the establishment by addressing it to the owner or manager. The surveys were distributed anonymously to each establishment. 
Each food establishment was mailed a notification letter one week before the survey was mailed out (Appendix A). The questionnaire was two pages on a single sheet of A4 paper and a letter accompanied the survey to describe the purpose of the survey (Appendix A). It was mailed out with a return self-addressed stamped envelope to return the questionnaire in. One week following the survey, a reminder letter was sent to each of the food establishments. The reminder letter included a web location where they could find the survey to print out, if they had misplaced the original survey. Contact information was included with each of the letters. However, none of the survey participants contacted me at anytime.

A total of 192 surveys were mailed out and we received a response from 62 participants. Three surveys were undeliverable, which resulted in a return rate of 33\%. The survey took place during December 2010, with all responses received within two months of mailing.

\subsubsection{Questionnaire design}

The questionnaire survey explored the attitudes of owners or managers of food establishments towards feral pigeons and measured their environmental knowledge, awareness, engagement and action (see Appendices for a copy of the questionnaire). The survey consisted of sixteen questions. The questions were a mix of open-ended and multiple-choice questions. The first part of the survey asked questions related to the participant's opinion on number of feral pigeons in Wellington City, how many and how often feral pigeons are seen at the food establishment, cost, what 
they do to discourage feral pigeons, and solutions they would support to manage feral pigeons.

The attitude of respondents towards feral pigeons was measured according to whether they thought there were too many pigeons in Wellington or the right number of pigeons in Wellington. Those that responded that there were too many pigeons in Wellington were perceived to have negative attitudes towards feral pigeons.

The second part of the survey focused on measuring environmental awareness, knowledge, engagement and action. Environmental awareness was measured by asking participants to state the three greatest threats to native plants and animals in New Zealand and matching these against expert opinion (Dowding and Murphy, 2001; Walker et al., 2006; Miskelly et al., 2008). Environmental knowledge was measured by asking participants to name eight bird species pictured. Environmental engagement was measured by asking participants to name three green spaces they most recently visited. And, environmental action was measured by asking participants to select activities that they have done in the last month/year, such as composting, recycling, and purchasing environmentally friendly products.

There were also demographic questions for age, sex, education, and ethnicity at the end of the survey. The categorisations followed those used in the last New Zealand census (2006). 


\subsubsection{Candidate models}

Hypotheses for how feral pigeons influence food establishment owners and managers' attitudes were weighted across a number of themes, including the cost of pigeons to their establishment, how frequently they see feral pigeons at their establishment, and whether pigeons receive food from their establishment.

I hypothesised that food establishments that frequently had feral pigeons at their establishment and who have to spend money to manage feral pigeons, may be more likely to think there are too many feral pigeons in Wellington City. Interactions with feral pigeons on a frequent basis and efforts to manage such interactions, may lead to negative attitudes towards feral pigeons.

I proposed that where feral pigeons obtain food from establishments, are seen frequently, and spend money to manage those interactions, food establishments may be more likely to have negative attitudes towards feral pigeons. However, it may also be the case that just seeing the pigeons and spending time cleaning up after the pigeons, may be a more significant indication of attitude towards feral pigeons.

I predicted that those who visit green space with indigenous species on a regular basis may be more likely to have negative attitudes towards feral pigeons. Persons who regularly visit green space may interact with native bird species and see feral pigeons in the city as displacing native species. Consequently, I hypothesised that people who only visit green space without habitat for indigenous species, such as city parks, may be 
more likely to have positive associations with pigeons.

For management of feral pigeons, we identified a number of questions that could potentially assist urban wildlife managers, including:

- Identification of preferred solutions to manage pigeons;

- Proportion of food establishments that have high numbers of pigeons;

- Proportion of food establishments that think there are too many feral pigeons;

- Main reasons food establishments think there are too many feral pigeons;

- Average cost of feral pigeons on food establishments;

- Proportion of food establishments where feral pigeons obtain food; and

- Main method for how pigeons obtain food from cafés.

The survey participants provided useful answers to these questions and are discussed below. Additional information can be requested from the authors.

\subsubsection{Statistical method}

I adopted an Information-Theoretic approach to testing hypotheses for measuring business attitudes towards feral pigeons. An Information-Theoretic 
approach examines several competing hypotheses simultaneously to identify the best set of models (i.e. hypotheses). The model with the best fit is the one that is best supported by the data (Johnson and Omland, 2004). The Information-Theoretic approach is distinguished from traditional null hypothesis by quantitatively ranking and weighting each competing hypothesis or model versus null hypothesis testing where an arbitrary probability threshold is used to support a hypothesis (Burnham and Anderson, 2002). The advantage of the Information-Theoretic method for our approach is that it allows the comparison of multiple, plausible models.

I generated a candidate set of hypotheses by using predictors of interest based on published literature and personal observation to derive submodels. Hypotheses were then characterised as logistic regression models (Keating and Cherry, 2004) using R2.13.0. The models were then compared using second order Akaike Information Criterion (AICc). The best models were selected by balancing improvement of model fit by adding parameters with a reduction in the quality of the parameter estimates by having less data per parameter (Posada and Buckley, 2004). To aid model selection, each model was ranked and weighted to provide a quantitative measure of relative support for each competing hypothesis. Models with $\triangle A I C c \leq 2$ were considered to have substantial support from the data (Burnham and Anderson, 2002). 


\subsection{Results}

The results are based on a total of 62 respondents from food establishment owners and managers. Most respondents were between 36 and 50 years old $(N=27,43 \%)$. The remaining respondents were evenly distributed amongst three age groups: $18-25(N=14,23 \%), 26-35(N=11,18 \%)$ and $51-70(N=10,16 \%)$. Education levels of respondents were high with most having at least a high school diploma and/or vocational degree and onehalf of respondents had either a bachelors or a post-graduate degree. The sex of respondents was nearly evenly split $(N=33$ females and $N=29$ males). Ethnicity of the sample was primarily New Zealand European $(N=38,61 \%)$ and the largest ethnic group was Asian $(N=12,19 \%)$. Four respondents identified as Maori and two respondents identified as Pacific Islander. Two respondents identified their ethnic group as other.

Feral pigeons are seen at food establishments mostly on a daily basis (Figure 4.2), but a substantial proportion seldom or never saw pigeons. When feral pigeons are present at food establishments, $56 \%$ of the time there are only $1-5$ pigeons present, $11 \%$ of the time there are $6-10$ pigeons present and $11 \%$ of the time there are 11-20 pigeons present. High numbers of feral pigeons (greater than 20 pigeons) are present only $20 \%$ of the time. The cost of feral pigeons on food establishments was reported by survey participants as low (Figure 4.1). Feral pigeons only receive/obtain food from $32 \%$ of the food establishments surveyed $(N=20)$. Where pigeons do obtain food from the establishment, they are most likely to get it from tables $(37 \%, N=10)$, floors $(18 \%, N=5)$, and patrons feeding $(18 \%$, 
$N=5$ ). Most food establishments reported spending less than $\$ 100$ per year, including labour costs (Figure 4.1). Only a small number of respondents spent quite a significant amount $(N=2$ spend over $\$ 5,000)$. Food establishment owners and managers stated the major problems with the feral pigeons in Wellington City are health risk, droppings, and nuisance.

Respondents supported aggressive feral pigeon control mechanisms by culling through methods such as poisoning $(N=43,69 \%)$ and shooting $(N=45,73 \%)$. Destruction of feral pigeon nests was also a popular control option $(N=39,63 \%)$. Less aggressive mechanisms such as regulations to prevent feeding of pigeons and littering of food waste received less support from food establishment owners and managers ( $N=25,40 \%)$ and $N=30,48 \%$, respectively).

Environmental knowledge scores for bird names were high with $66 \%$ of respondents scoring between 5-8 out of 8. Over half of respondents (53\%) visited green space at least weekly and they most often visited suburban parks. Only one survey participant had recently visited a park outside the city. Environmentally friendly behaviors or actions were common: $85 \%$ of respondents recycled $(N=53) ; 77 \%$ offered environmentally friendly products for purchase $(N=48) ; 46 \%$ composted $(N=29)$; and $40 \%$ donated to environmental causes $(N=25)$.

\subsubsection{Model Results}

The candidate model results showed that three variables have the primary influence on food establishment owner/managers' attitudes to feral pi- 
geons (Table 4.1). The cost of feral pigeons on food establishments (Figure 4.1) and the frequency that pigeons are seen at food establishments Figure 4.2) had the most significant influence on café owner attitudes ( $\triangle \mathrm{AIC} \leq 2$, $W=0.465)$. Whether feral pigeons obtain food from the establishment also plays a role in influencing attitudes $(\triangle \mathrm{AICc} \leq 2, W=0.190)$ (Figure 4.3).

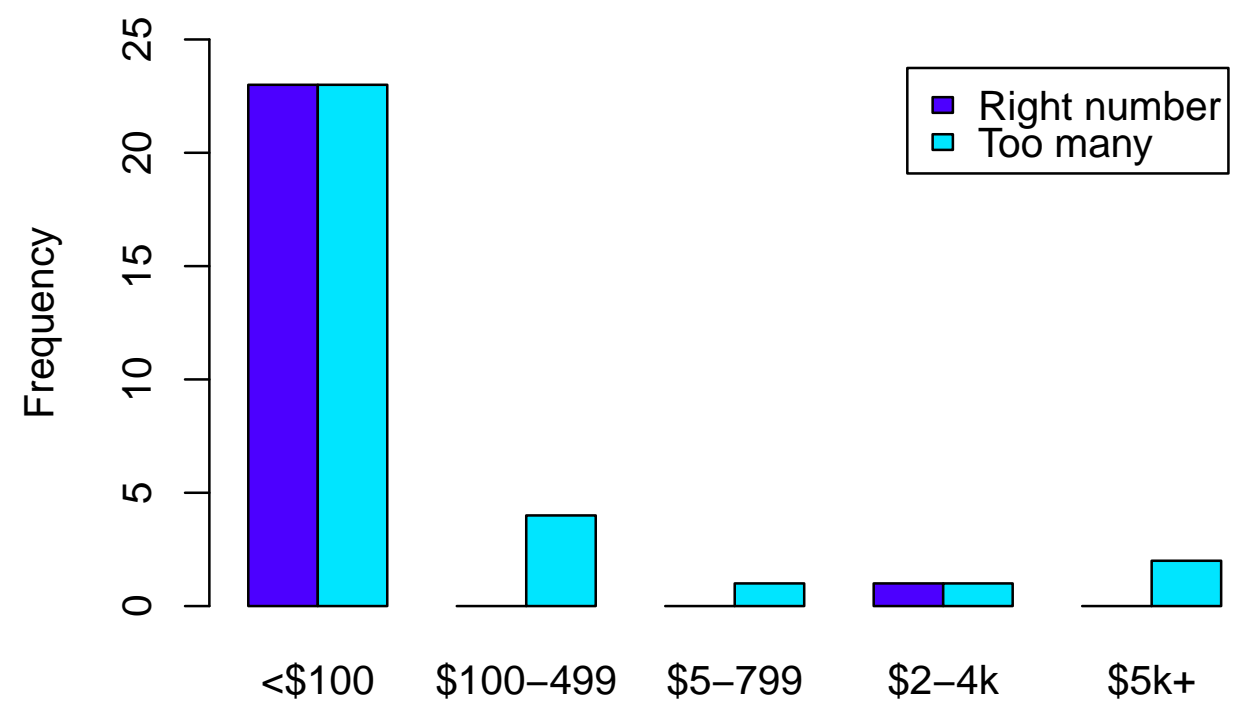

Amount spent to deter pigeons

Figure 4.1: Attitude of respondents to feral pigeons and the annual cost of feral pigeons to food establishments $(n=62)$.

A confidence set of socio-demographic variables (age, (Figure 4.4), sex (Figure 4.5) and education (Figure 4.6)) showed that demographics were 


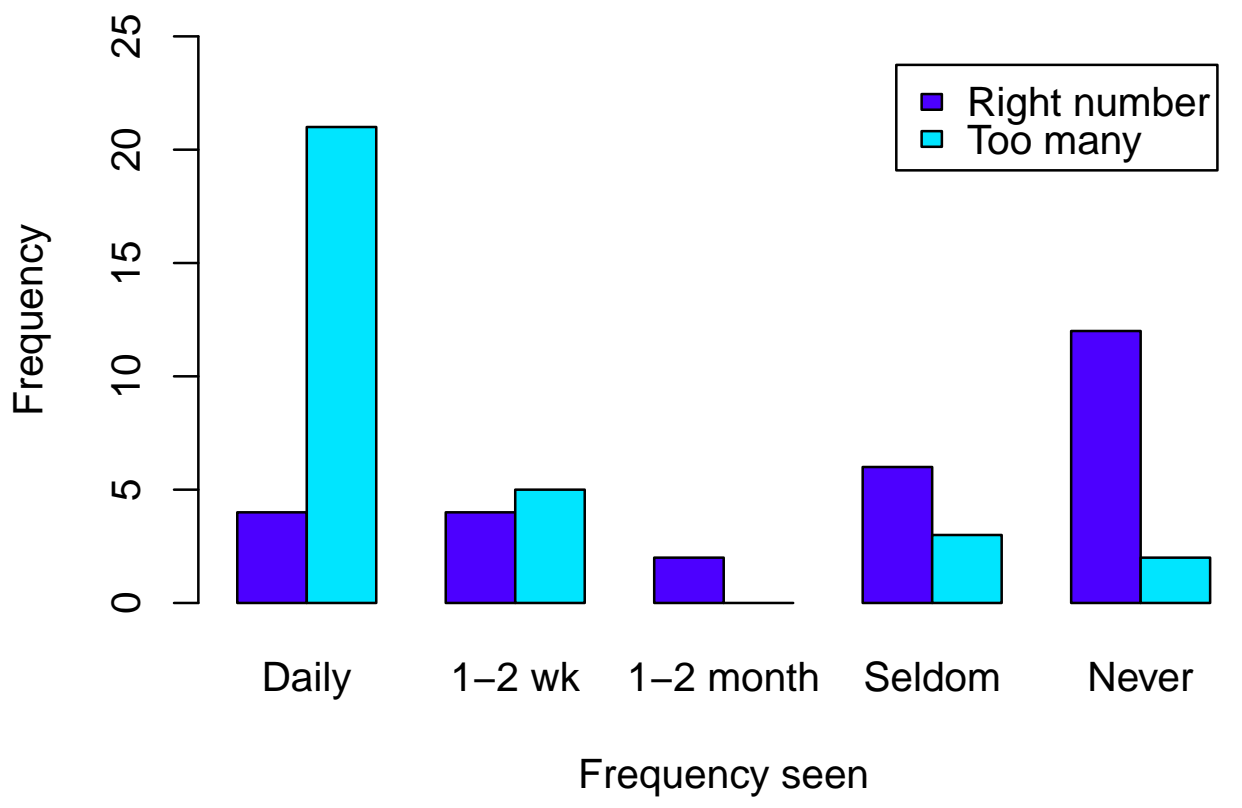

Figure 4.2: Attitude of respondents to feral pigeons and the frequency feral pigeons were seen at food establishments $(n=62)$. 


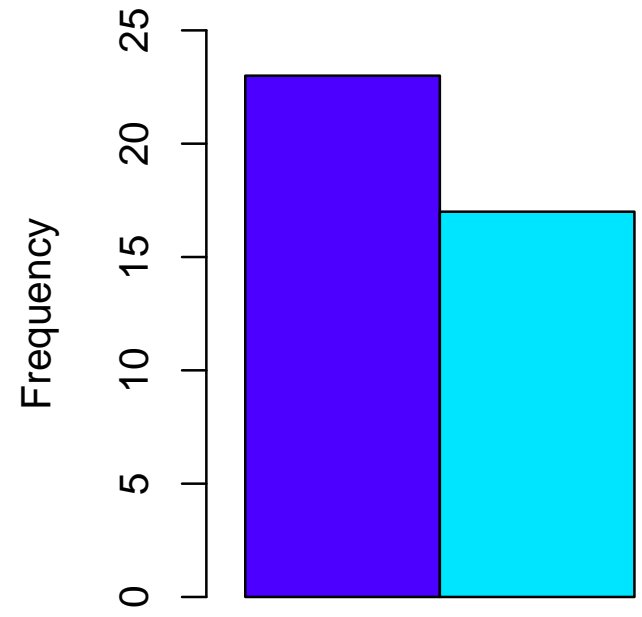

No
- Right number Too many

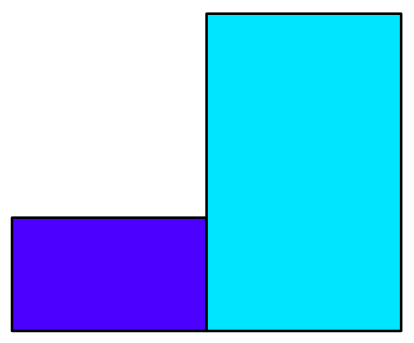

Yes

Do pigeons obtain food from establishment?

Figure 4.3: Attitude of respondents to feral pigeons and the frequency feral pigeons obtain or receive food from establishments $(n=62)$. 


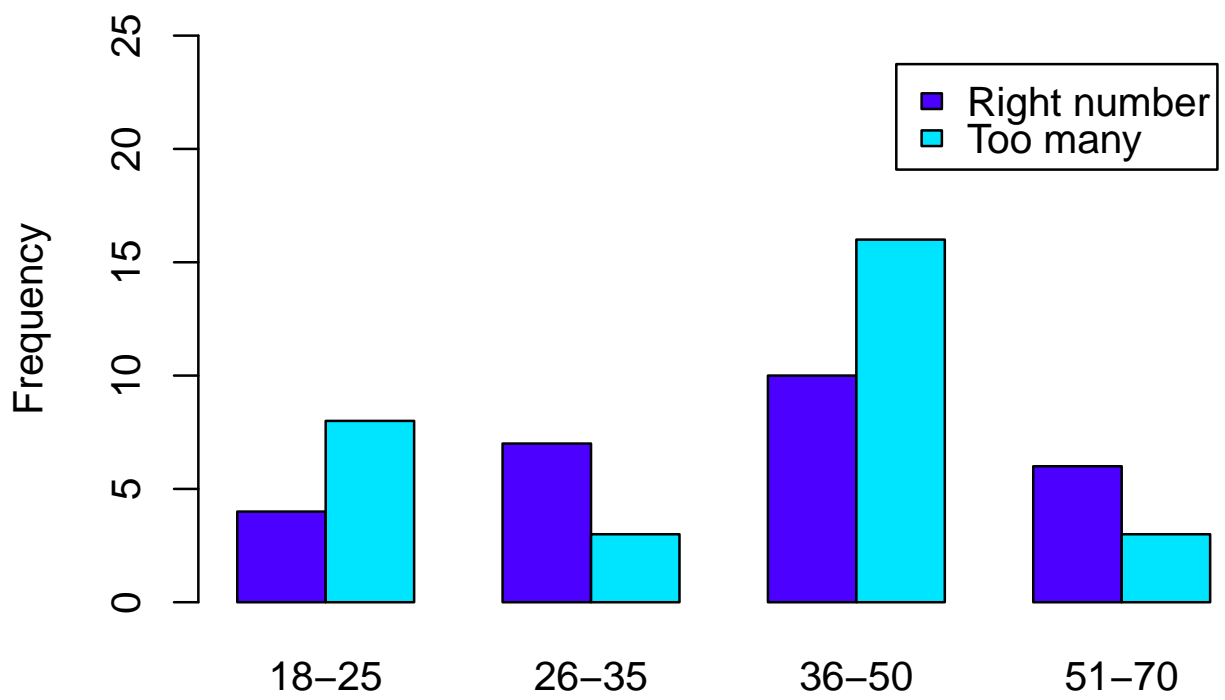

Figure 4.4: Attitude of respondents to feral pigeons on number of pigeons by age of respondents $(n=62)$.

associated with the results to some extent, but did not influence people's attitudes (Table 4.2). No conclusions were based on ethnicity because of the small sample size of minority ethnicities $(N=18)$. 
Table 4.1: Candidate models with socio-demographic variables (age, sex and education variables are indicated by ${ }^{* * *}$ )

\begin{tabular}{|c|c|c|c|c|}
\hline Models & $k$ & AICc & $\Delta \mathrm{AICc}$ & Akaike $w_{i}$ \\
\hline Cost, frequency seen & 3 & 59.208 & 0 & 0.465 \\
\hline Cost, frequency seen, ${ }^{* * *}$ & 6 & 60.911 & 1.703 & 0.198 \\
\hline Food from establishment, frequency seen, cost & 4 & 61.004 & 1.796 & 0.189 \\
\hline Food from establishment, frequency seen, cost, $* * *$ & 7 & 62.757 & 3.549 & 0.079 \\
\hline Frequency seen, ${ }^{* * *}$ & 5 & 63.457 & 4.248 & 0.056 \\
\hline Frequency seen & 2 & 66.335 & 7.127 & 0.013 \\
\hline Cost & 2 & 74.279 & 15.071 & 0.000 \\
\hline Cost, $* * *$ & 5 & 77.864 & 18.656 & 0.000 \\
\hline Food from establishment & 2 & 80.663 & 21.455 & 0.000 \\
\hline Food from establishment, $* * *$ & 5 & 81.318 & 22.110 & 0.000 \\
\hline Knowledge & 2 & 84.792 & 25.584 & 0.000 \\
\hline Awareness, knowledge & 3 & 85.277 & 26.069 & 0.000 \\
\hline Engagement & 2 & 86.047 & 26.838 & 0.000 \\
\hline Engagement, ${ }^{* * *}$ & 5 & 87.315 & 28.106 & 0.000 \\
\hline Knowledge, awareness, action & 4 & 87.338 & 28.130 & 0.000 \\
\hline Awareness, knowledge, engagement & 4 & 87.537 & 28.329 & 0.000 \\
\hline Awareness, action & 3 & 87.702 & 28.493 & 0.000 \\
\hline Knowledge, $* * *$ & 5 & 87.794 & 28.586 & 0.000 \\
\hline Engagement, action & 3 & 87.857 & 28.649 & 0.000 \\
\hline Awareness, knowledge, $* * *$ & 6 & 89.104 & 29.895 & 0.000 \\
\hline Engagement, action, ${ }^{* * *}$ & 6 & 89.686 & 30.477 & 0.000 \\
\hline Awareness, action, $* * *$ & 6 & 89.927 & 30.719 & 0.000 \\
\hline Awareness, knowledge, engagement, $* * *$ & 7 & 91.260 & 32.052 & 0.000 \\
\hline Knowledge, awareness, action, $* * *$ & 7 & 91.510 & 32.302 & 0.000 \\
\hline
\end{tabular}


Table 4.2: Attitudes of food establishments towards feral pigeons

\begin{tabular}{lllcc}
\hline Demographic models & $k$ & AICc & $\Delta$ AICc & Akaike $w_{i}$ \\
\hline Age & 2 & 82.280 & 0.000 & 0.221 \\
Sex & 2 & 82.480 & 0.200 & 0.200 \\
Education & 2 & 82.720 & 0.440 & 0.177 \\
Age + Education & 3 & 84.060 & 1.780 & 0.091 \\
Age + Sex & 3 & 84.080 & 1.800 & 0.090 \\
Education + Sex & 3 & 84.380 & 2.100 & 0.077 \\
Sex + Age + (Sex * Age) & 4 & 85.904 & 3.624 & 0.036 \\
Age + Education + Sex & 4 & 85.920 & 3.640 & 0.036 \\
Age + Education + (Age * Education) & 4 & 86.537 & 4.257 & 0.026 \\
Sex + Education + (Sex * Education) & 4 & 87.151 & 4.871 & 0.019 \\
Sex + Age + Education + (Sex * Age) & 5 & 87.991 & 5.711 & 0.013 \\
Sex + Age + Education + (Education * Age) & 5 & 88.853 & 6.573 & 0.008 \\
Sex + Age + Education + (Education * Sex) & 5 & 89.084 & 6.804 & 0.007 \\
\hline
\end{tabular}



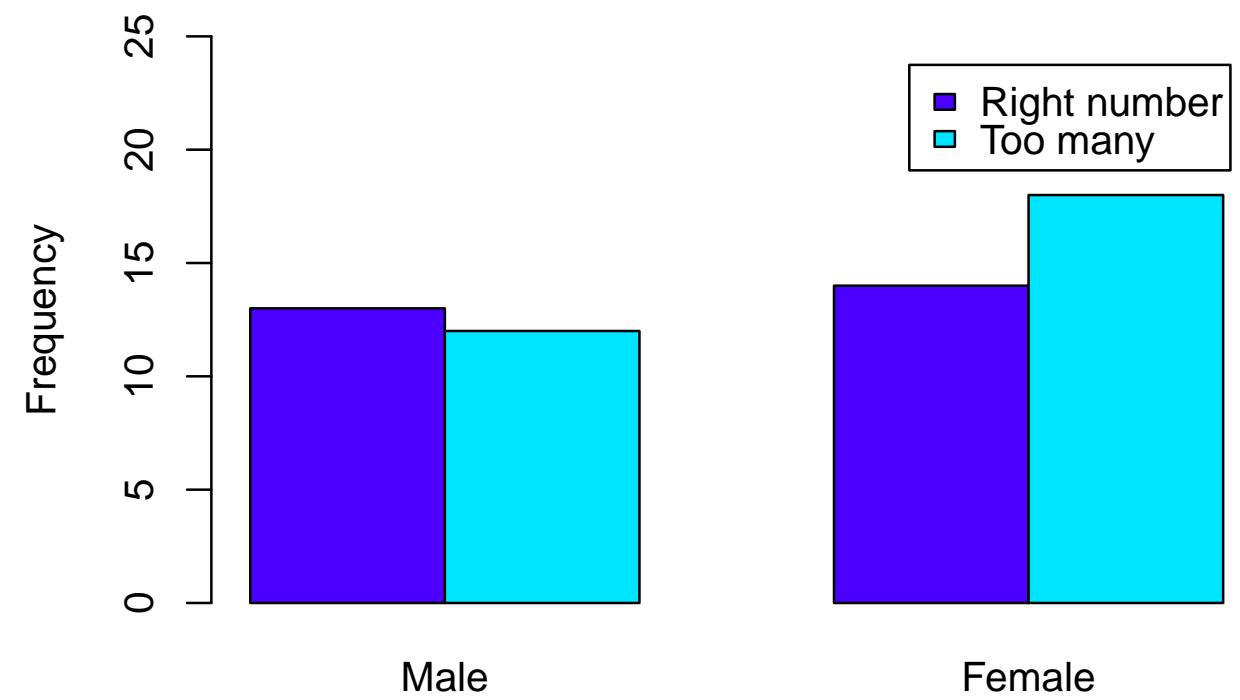

Figure 4.5: Attitude of respondents to feral pigeons by sex of respondents $(n=62)$. 


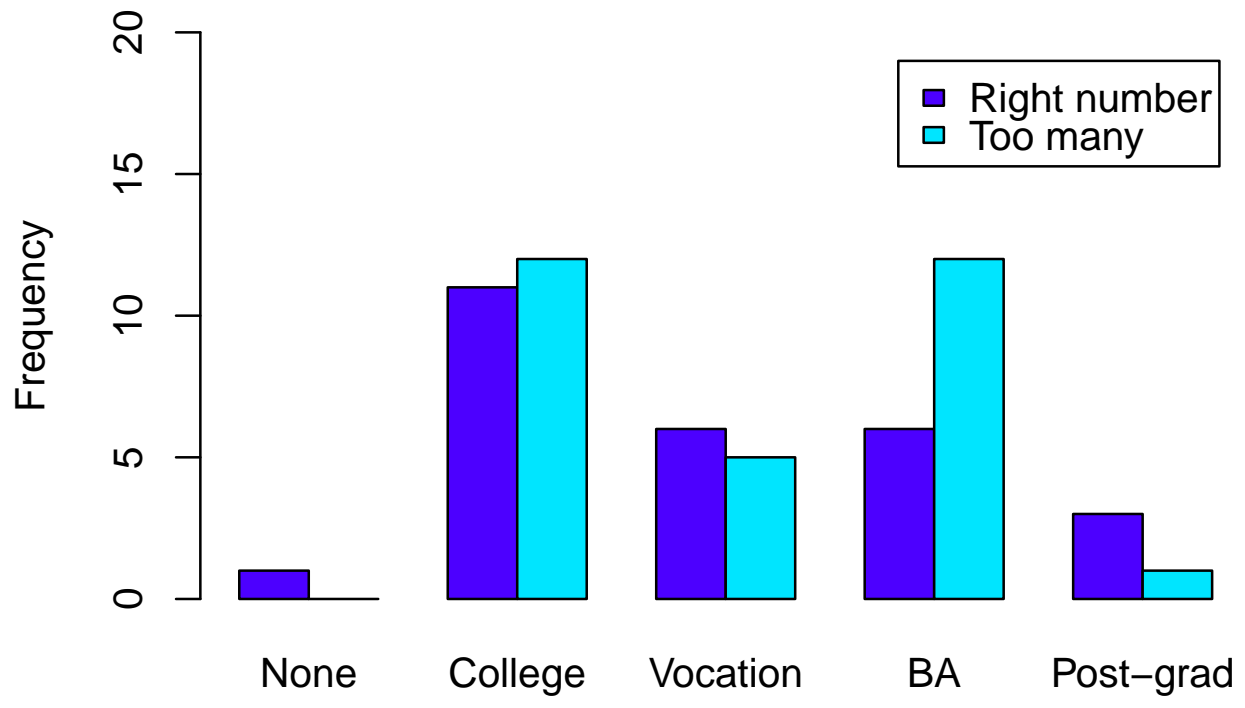

Figure 4.6: Attitude of respondents to feral pigeons by education level of respondents $(n=62)$. 


\subsection{Discussion}

Feral pigeons are frequently seen at food establishments in low numbers. Most food establishments do not have large numbers of feral pigeons at their sites, but small groups of 1-5 pigeons are the most common. Food establishments are not spending large amounts of money to control feral pigeon problems - the majority report spending less than $\$ 100$ every year and we suspect many food establishments spend nothing (the survey did not have an option for participants to select $\$ 0)$.

Feral pigeons appear not be getting food from food establishments. This supports the hypothesis that people are directly feeding pigeons (see Chapter 2 herein). But, where feral pigeons are getting food from establishments, they mostly get food from tables. This indicates that food establishments should focus on clearing tables to deter feral pigeons from getting food from their establishment.

Despite food establishments not having to spend money to deter pigeons or even see many of them at their establishment, owners and managers still think there are too many feral pigeons in Wellington City. This indicates that even a small number of pigeons that regularly visit a particular place can have a large impact on people's attitudes. It also shows that the cost of feral pigeons is not only monetary. Food establishments stated the major problems with the feral pigeons in Wellington City are health risk, droppings, and nuisance - none of which are directly related to the financial cost of pigeon damage. Food establishments are probably concerned about the health risk of pigeons and their droppings in their es- 
tablishments as well as the nuisance to customers, e.g., displaying begging behaviour and getting underfoot.

To manage feral pigeons in Wellington, food establishments show a high tolerance to aggressive feral pigeon management. High numbers of food establishments support poison and culling as management options. In contrast, the wider Wellington population shows support for regulation and education programmes that focus on responsible feeding of wildlife (culling and poisoning were the least popular methods of control) (Ryan, 2011). The difference in attitude between food establishments and residents demonstrates the importance that regular interaction and cost can have on people's attitudes. Additionally, any pigeon management should investigate the cause of the conflict because people's attitudes vary depending on the situation. The results indicate that different members of the community will support significantly different management techniques, which could cause conflict between groups of people within the same society.

\subsubsection{Attitudes}

The most significant result is that immediate experience of the attitude object (feral pigeons) shapes attitudes more strongly than (1) socio-demographics;

(2) knowledge; and (3) engagement.

The leading model indicates that people's attitudes to feral pigeons are most likely influenced by how often they see feral pigeons ( $\triangle \mathrm{AIC} \leq 2$, $W=0.465)$, with more frequent interactions resulting in a negative atti- 
tude. These findings are consistent with a study on environmental conditions and attitude, which found that environmental conditions shape attitudes (Johnson et al., 2005). This has important policy implications because it suggests that policy outcomes are more important than just adopting new policies. In the feral pigeon context, a local authority's campaign to reduce feral pigeon populations may not be effective unless it also reduces the frequency that individual food establishments see feral pigeons.

A reduction in the frequency that businesses encounter pigeons, may change attitudes in a positive manner. Thereby, city officials do not need to also address changing people's attitudes directly because any pigeon management will also influence people's attitudes. Because food establishment owners and managers' attitudes are dynamic and shaped by direct experience, it will be easier for urban wildlife managers to address any negative attitudes towards wildlife than it would be if attitudes were static. If city officials can control feral pigeons, it may result in fewer negative attitudes.

The cost of feral pigeons on food establishments also features in the other two leading models, but not on its own. The results indicate that even a slight increase in the cost for food establishments increases the likelihood that they will have negative attitudes towards feral pigeons. Therefore, it is essential that any feral pigeon management does not increase the costs on business owners.

Whether pigeons receive food from the establishment also influences people's attitudes towards feral pigeons. If feral pigeons receive or obtain 
food from establishments, the owners and managers of the food establishment are more likely to have a negative attitude towards feral pigeons. This supports the conclusion that pigeons getting food from people is the greater problem (see Chapter 2 herein).

The socio-demographic variables do not improve the fit of the leading models (Table 4.2). Attitudes are better described by direct, less abstract, characters of people. This adds further support to the results that environmental experience is the primary influence on attitudes. The lack of explanation of behaviour based on demographic variables has been supported in other studies. Indeed, some studies recommend focusing on variables other than demographics, which have been given too much emphasis in the past, to explain behaviour (Straughan and Roberts, 1999). My results support this contention.

A number of the variables, such as environmental knowledge and action, do not influence business owners and managers' attitudes towards feral pigeons. People's environmental knowledge, such as effects of introduced species, may not change people's attitudes towards pigeons. Environmental education may not be as powerful as direct experience in influencing people's attitudes. This is an important result for environmental education, which these results support including a direct experience component. Therefore, urban pigeon management should likely focus on preventing people from feeding rather than educating people about the effects of feral pigeons. Increased knowledge of feral pigeons is less likely to change people's attitudes towards feral pigeons. 
Overall business attitudes are incredibly informed by their direct experience with pigeons - it is not just a general anti-pigeon attitude. Those who interact with pigeons less often are less likely to hold negative views of feral pigeons. In addition to the cost on food establishments, the conflict may stem from the deterrence of customers who may associate feral pigeons with health risks, droppings and general annoyance. This cost may not have been calculated by business owners and managers when estimating the costs of feral pigeons on their establishment.

It is important to address negative attitudes towards urban wildlife because of the Pigeon Paradox (Dunn et al., 2006). The Pigeon Paradox suggests that people's negative attitudes towards urban wildlife could effect their willingness to participate in conservation initiatives (Dunn et al., 2006). Therefore, despite the low cost of feral pigeons on food establishments, the negative attitudes of business owners could have other impacts on society, particularly in relation to conservation agendas. There is substantial literature demonstrating that environmental attitudes significantly influence environmentally-related behavioural intentions and behaviour (Fransson and Gärling, 1999). And, the public's involvement in environmental issues should be used as a great resource to tackle conservation problems (Caula et al., 2009).

Overall, the greater problem is feral pigeons receiving food from people (as discussed in Chapter 1 and 2 herein), with approximately 20\% of bird feeders in Wellington feeding pigeons (Ryan, 2011). Experience drives the attitudes of owners and managers of food establishments. This re- 
sult demonstrates that the problem of negative attitudes of business owners and managers can be managed by reducing the number of pigeons. Business owners are important figures in society because of their ability to influence the community and fund projects and their support in conservation initiatives would be an advantage. The key to urban wildlife management is to change people's behaviour to limit feeding, which will likely result in a change in negative attitudes of business owners. 


\section{Chapter 5}

\section{Conclusion}

Urban ecology is important to the field of ecology because of the significant growth of urban populations and expansion of modified landscapes. There is a need to understand how animals exist in cities in order to manage their populations and relationships with people. Most of the world's human population will live in cities soon. In New Zealand, almost threequarters of people live in main urban areas (Statistics New Zealand, 2006). The shift to urban populations requires a fundamental change in urban wildlife management and decision-making that involves the public (Patterson et al., 2003). People's perspectives on wildlife are highly individualised which increases the likelihood of social conflict in cities over wildlife management (Patterson et al., 2003).

I found that one of the most successful colonisers of urban areas - feral pigeons - are attracted to sites where people congregate to eat. Pigeons are more likely to be in areas where people congregate to eat, such as areas 
with outdoor seating, than in other areas of the City. People's direct feeding is most likely supporting higher levels of feral pigeons in Wellington City. This highlights the special relationship between people and wildlife and the importance of people in the study of urban ecology.

The results indicate that feral pigeons may not be food limited in central Wellington City. Anthropogenic food appears to be plentiful. Food quantity at sites was based on what was left behind by the pigeons. Indeed, we observed uneaten food at all sites, which is more likely to indicate a satiated population rather than unexploited food. The consistency of feral pigeon condition and activity levels across the sites suggests that food is either sufficient to satiate all individuals at each site (even those with low amounts of artificial food) or some are travelling to other sites to feed. Given that other work suggests the movements of pigeons are small distances (Ryan, 2011), the former is likely to be true of most of the population. Direct feeding of wildlife may affect numerous urban wildlife species and indeed affect the larger urban ecosystem. Urban ecology studies should include consideration of people and how direct feeding of wildlife can alter urban wildlife populations.

Urban wildlife conflicts can be higher with particular members of the community and result in polarised communities that urban authorities struggle to manage. Studies of human-wildlife conflicts should pay particular attention to food establishments in understanding the human dimension of conflict. Owners and managers of food establishments play a special role with urban wildlife management issues because they may be 
more likely to have interactions with urban wildlife because of food availability and disposal. And, many of those interactions may be negative. Café and restaurant owners are also key members of the business community with the influence to instigate pigeon control measures. This study highlights the need to consider that urban wildlife conflicts can have different origins for different people. It also shows that urban ecology studies should include people that are directly affected by the particular species of interest.

Overall business attitudes are incredibly informed by their direct experience with pigeons. It is not just an anti-pigeon attitude generally. The conflict is likely to stem from the cost of feral pigeons on food establishments and possibly from the deterrence of customers who associate feral pigeons with health risks and nuisance. This result creates a number of urban wildlife management recommendations, which are discussed below. But, it is also important to note that this is likely to be the case for any human-wildlife conflict and should be taken into account when considering management options.

Environmental knowledge and awareness do not appear to influence business owners and managers' attitudes towards feral pigeons. As a result, it may not be worthwhile increasing people's environmental knowledge, such as education on the effects of introduced species, to change people's attitudes towards pigeons. Environmental education is less likely to be as powerful as direct experience in influencing people's attitudes. This is an important result for urban wildlife management because these 
results support human-wildlife conflict resolution options that are based on direct experience - rather than education alone because provision of information is not enough to change people's attitudes.

Food establishment owners and managers' attitudes towards feral pigeons were mostly negative, which could have far reaching effects on communities and beyond. Negative attitudes to feral pigeons could result in a polarised community with conflict over management options of feral pigeons. Negative attitudes can also have consequences beyond the immediate community to conservation generally. Business leaders' influential positions in the community and their financial position means that their attitudes towards wildlife are important for the success of conservation initiatives.

With the majority of people living in cities, conservation initiatives may now depend on people who live in cities. As a result, the success of conservation initiatives may be linked to people's interactions with urban wildlife (Dunn et al., 2006). Urban residents' interactions with wildlife may be limited to introduced species or other successful urban colonisers. These urban colonisers are often the "wrong" type of wildlife to form attachments to. Also, urban residents are more likely to suffer the annoyances of urban wildlife and may have negative associations with wildlife. If urban residents have negative attitudes towards urban wildlife, such as feral pigeons, it may influence their willingness to participate in conservation initiatives. This could have wide ranging effects in the future because of the increase of people living in urban areas. The management of urban 
wildlife needs to mitigate negative experiences of people.

\subsection{Management implications}

The most effective management solution or tool is most likely to be limiting direct feeding of pigeons by people. Education alone is unlikely to be enough to result in a change of behaviour. By modifying the amount of food that people provide to feral pigeons, pigeon populations are likely to decrease. Indeed, one study found that by cleaning spillage of wheat and grain reduced pigeon populations (Murton et al., 1972a). And, by reducing the number of pigeons, it may result in improved attitudes towards feral pigeons.

A consistent approach across sites in Wellington is necessary because feral pigeons do not appear to be food limited at particular sites. Control of anthropogenic sources of food will need to be broad-based. A landscapescale or city wide strategy is necessary because control of food at one site, may only cause feral pigeons to move to other sites for food and may not influence population size.

By focusing on areas where people are likely to congregate to eat, it allows for city authorities to put more effort into educating the public in those areas through social marketing and incentives (not just the provision of information as increased knowledge of the problem may have little effect on people's attitudes). Signs instructing people not to feed pigeons may not be particularly helpful as it is difficult to monitor (labour inten- 
sive option for cities) and enforce (heavy fines may not be politically viable as much of the city enjoys bird feeding) (Ryan, 2011), and any monitoring is likely to be costly on city authorities. Education on the effects of pigeon feeding, e.g., increasing population levels to a point that cause conflict with people, needs to be seriously considered - potentially with a direct experience component.

Increasing incentives on the public not to feed pigeons and increasing efforts to contain food waste is likely to be more cost effective and durable than ongoing removal of feral pigeons or maintenance of feral pigeon lofts. Addressing pigeon problems by changing people's feeding behaviour meets animal welfare concerns for wildlife management. It is also a much more politically viable option than culling, which often gathers much public opposition. Also, culling has been shown to have limited success in the ongoing management of feral pigeons. Studies have shown that new immigrants will take the place of those culled and the population will rise to the same level as before the culling operation (Sol and Senar, 1995). Pigeons from other areas will replace removed birds within a short period of time (Rose et al., 2006). Additionally, culling is very expensive and requires continual removal of birds for it to be effective (Murton et al., 1972a). It is unlikely to be a viable option for many cities because of the substantial cost for limited term effects. Studies have shown that management costs can be as high as nine dollars a pigeon per year (HaagWackernagel, 1995). Central city feral pigeon population may be as dense as 6.8 pigeons per hectare in Wellington City, thus making culling opera- 
tions financially prohibitive (Ryan, 2011).

Other direct control management options include removal of eggs from nesting areas (Haag-Wackernagel, 1995), and provision of birth control (Dobeic, 2003). Each of these methods have indicated limited success. The provision of birth control through hormonally treated maize resulted in a 24.3 per cent decrease of feral pigeons across three years in Ljubljana, Slovenia (Dobeic, 2003). Using birth control is a very resource intensive method of control and required daily feeding of pigeons during mating seasons. As a result, this method may not be achievable for most cities.

The method used in Basel, Switzerland involved setting up a loft for feral pigeons (Haag-Wackernagel, 1995). Wildlife managers regularly remove eggs from pigeons' nests and replace them with plastic eggs. Pigeon lofts meet animal welfare concerns and are popular with the general public in Basel, Switzerland. It allows for the public to enjoy having a limited number of healthy feral pigeons in their town. This method is also resource intensive and costly and can meet with limited success in areas where there is not a shortage of nesting spots. This study indicates that feral pigeons in Wellington City are not limited in the availability of roosting and nesting spots (see Chapter 2 herein) and therefore, this method may have limited success in Wellington City.

A public education approach to feral pigeon management is a longterm strategy, i.e., will not be immediately effective as education and change in behaviour will take time, that is likely to be cost-effective. Feral pigeon management in Wellington City is not an immediate concern as indicated 
by the low cost on food establishments. Although many food establishments hold negative views towards pigeons, they are not very expensive for the majority of food establishments and there is limited effect on native species or the environment generally. And, by limiting people's feeding behaviour and a resulting population decline, the attitudes of food establishments will likely improve. There is no need to also work on changing peoples attitudes as the modification of the pigeon population will most likely result in a change of attitude because attitudes were closely linked to experience.

These management recommendations could be applied in other cities with feral pigeon conflicts. It may be particularly applicable to those cities that have found culling and pigeon lofts unsuccessful. The lack of success of culling and lofts may indicate that artificial foods are driving populations. If people keep their food inaccessible and limit any direct feeding to feral pigeons, it may provide a management solution for feral pigeon problems in cities around the world.

Further research is needed on how education (e.g., the right incentives) can change people's wildlife feeding behaviour. People support pigeon populations at levels that are artificially high by directly feeding pigeons. Is this a signal that people are forming connections with the "wrong" type of wildlife? Or, can education of the public help direct peoples' interactions with wildlife in a more positive manner? Research on effective education methods that include a direct experience component should be carried out, particularly since signs appear to have little effect on people 
feeding pigeons (based on personal observation over a year). 


\section{Appendix A}

\section{Survey samples}

Below are copies of the survey and letters referenced in Chapter 4. 


\section{Urban wildlife management and feral pigeons in Wellington}

\section{PLEASE START HERE}

1. How many feral pigeons do you see at your business and how often?
$\square \quad 1-5$ pigeons
$\square$ 6-10 pigeons
$11-20$ pigeons
$\square \quad 21-30$ pigeons
$\square \quad 31-50$ pigeons
$50+$ pigeons

$\square$ Daily

$\square$ Once or twice a week

$\square$ Once or twice a month

$\square$ Seldom

$\square$ Never

2. Do feral pigeons get food from your business? $\square$ Yes $\square$ No. If yes, please specify:

$\square$ Tables

$\square$ Rubbish bins or skips

$\square$ Patrons feeding

3. In your opinion, in Wellington there are:

Too many feral pigeons

$\square$ Not enough feral pigeons

$\square$ About the right number of feral pigeons

$\square$ No opinion about number of feral pigeons

$\square$ Store/delivery room

$\square$ Other:
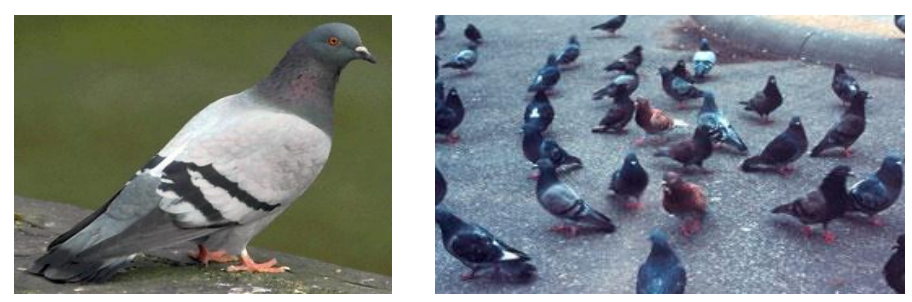

4. Please explain why you chose your answer in question 3.

5. What does your establishment do to discourage feral pigeons?

6. Please estimate what feral pigeons cost your business annually in expenses and lost earnings:
$\square$ Less than $\$ 100$
$\square \$ 100-\$ 499$
$\$ 500-\$ 799$
$\$ 800-\$ 1,499$
$\$ 1,500-\$ 1,999$
$\$ 2,000-\$ 4,999$
$\square \$ 5,000+$

7. What solutions would you support to manage feral pigeons in Wellington? Check as many that apply.

$\square$ Poisoning pigeons

Shooting pigeons

Destruction of pigeon nests on private and public property

$\square$ Regulation for barriers to prevent pigeons from using private and public buildings to nest and roost

$\square$ Regulation to prevent pigeons from feeding on food waste from rubbish bins and skips

$\square$ Regulation to prevent the public from feeding pigeons

$\square$ Other (please specify):

8. How often did you visit a green space (park, reserve, or public gardens) in the last year?

Once a week

Two to three times a week

$\square \quad$ Two to three times a month

$\square \quad$ Once a month

$\square \quad$ Once or twice a year

$\square \quad$ Not at all

9. Name three green spaces (parks, reserves, or public gardens) that you most recently visited: i. ii. iii. 
1. Write the name of the bird species pictured* in the blank space provided, or tick the 'don't know' box if unsure.

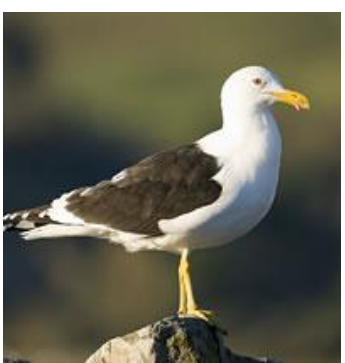

Bird 1

$\square$ Don't know

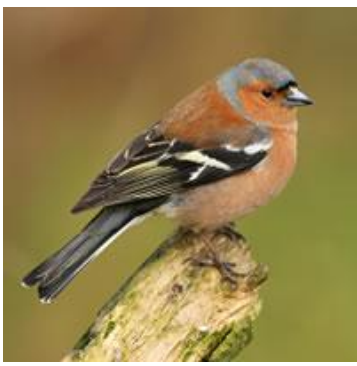

Bird 5

$\square$ Don't know

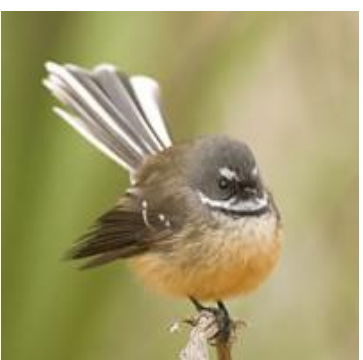

Bird 2

$\square$ Don't know

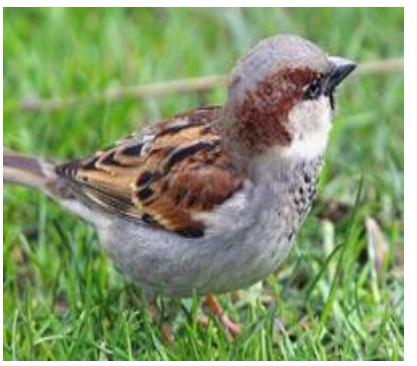

Bird 6

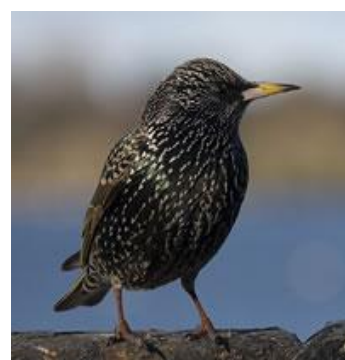

Bird 3

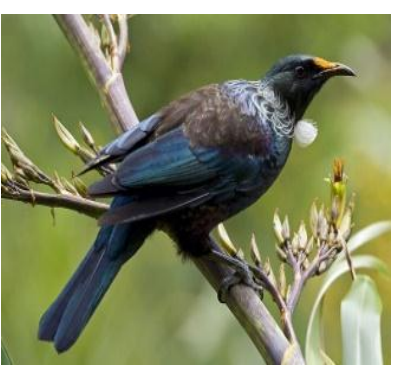

Bird 4 $\square$ Don't know

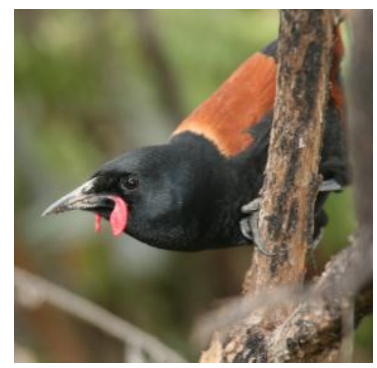

Bird 7 $\square$ Don't know

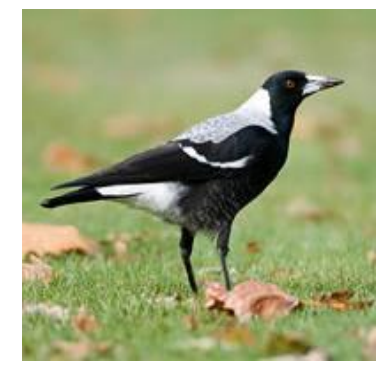

Bird 8

$\square$ Don't know

2. What do you think are three greatest threats to native plants and animals in New Zealand?

i. ii. iii.

\begin{tabular}{|l|c|c|}
\hline 12. How many of these activities have you done in the last month? & Yes & No \\
\hline Composting organic waste & $\square$ & $\square$ \\
\hline Recycling & $\square$ & $\square$ \\
\hline Purchase environmentally friendly products or food & $\square$ & $\square$ \\
\hline How many of these activities have you done in the last year? & Yes & No \\
\hline Donated to an environmental charity & $\square$ & $\square$ \\
\hline Volunteered for an environmental organisation & $\square$ & $\square$ \\
\hline Attended a public meeting in support of the environment & $\square$ & $\square$ \\
\hline Signed a petition in support of the environment & $\square$ & $\square$ \\
\hline
\end{tabular}

13. Are you:
$\square$ Female
$\square$ Male

14. Please tick $\square$ the age group you belong to.
$\square 18-25$
$\square 26-35$
$\square 36-50$
$\square 51-70$
$\square 70+$

15. What is your highest educational level?

$\square$ No formal qualifications

$\square$ High school qualification

$\square$ Vocational/trade qualification

$\square$ Bachelor degree

$\square$ Postgraduate degree

16. Which ethnic group do you belong to:
$\square$ New Zealand European
$\square$ Maori
$\square$ Pacific Islander
$\square$ Asian
$\square$ Other. Please specify 
VICTORIA UNIVERSITY OF WELLINGTON

Te Whare Wānanga o te Ūpoko o te Ika a Māui

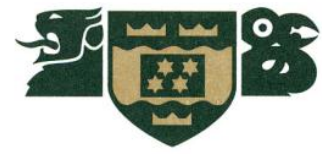

Participant Information Sheet

Research Project:
"Urban wildlife management and feral pigeons in Wellington"

$8^{\text {th }}$ November 2010

Researcher: Symone Krimowa

Telephone (mobile): 0211408967

Email: symonekrimowa@gmail.com
Supervisor: Dr Wayne Linklater

Telephone: 044635233

Email: wayne.linklater@vuw.ac.nz
School of Biological Sciences Victoria University of Wellington PO Box 600

Wellington 6140

\title{
Dear Owner/Manager,
}

I am writing to ask your help in a study that is part of a master's degree which is being conducted to investigate the relationship between urban wildlife such as feral pigeons and local businesses. Results from this study will help better understand wildlife in cities and has important implications for city planning.

Your business has been chosen due to its geographic location. I am inviting the business owner or manager to participate in a questionnaire that will not exceed ten minutes in length ( 2 pages).

The information on the questionnaire is anonymous; you will not be asked your name or other sensitive information. You are provided with a self-addressed stamped envelope so that you can send the completed questionnaire anonymously to School of Biological Sciences, Victoria University of Wellington, PO Box 600, Wellington 6140. The results of the questionnaire will only be used for our study. This survey is voluntary and has been approved by the Human Ethics Committee. Returning the survey implies consent for participation in this study. We hope you will take a few minutes to help our research by filling in the questionnaire.

If you have any other questions about this project, please feel free to contact me from the contact details provided above. Results will be available on this website: http://www.victoria.ac.nz/sbs/staff/wayne-linklater.aspx. A copy of the survey is also available on the website. We will send a reminder letter shortly. If you have already returned the questionnaire, please ignore the reminder letter.

Thank you very much for helping with this important study.

Sincerely,

Symone Krimowa

MSc candidate, Victoria University of Wellington

\author{
SCHOOL OF BIOLOGICAL SCIENCES \\ Te Kura Mātauranga Koiora \\ FACULTY OF SCIENCE \\ Te Wāhanga Pūtaiao \\ P.O. Box 600, Wellington, New Zealand \\ Telephone +64-4-463-5339, Facsimile +64-4-463-5331 \\ Email Biosci@vuw.ac.nz
}


VICTORIA UNIVERSITY OF WELLINGTON

Te Whare Wānanga o te Ūpoko o te Ika a Māui

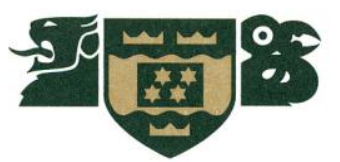

Urban wildlife management and feral pigeons in Wellington

$1^{\text {st }}$ November 2010

Dear Owner/Manager,

About a week from now you will receive in the mail a request to fill out a brief questionnaire designed to measure the effects of feral pigeons on local businesses in Wellington. Your business has been chosen due to its geographic location in central Wellington.

The questionnaire will provide information for an important research project being conducted as part of a Master's degree at Victoria University of Wellington, New Zealand.

I am writing in advance because many people like to know ahead of time that they will receive the survey. Your answers on the questionnaire are confidential and anonymous. The results of the questionnaire will only be used for our study. Once the study is complete, a report will be made available to you and all other participants.

Thank you for your time and consideration with this important research. It is only with the generous help of people like you that our research can be successful.

Sincerely,

Symone Krimowa

MSc candidate, Victoria University of Wellington

\section{SCHOOL OF BIOLOGICAL SCIENCES}

Te Kura Mātauranga Koiora

FACULTY OF SCIENCE

Te Wāhanga Pūtaiao

P.O. Box 600, Wellington, New Zealand

Telephone +64-4-463-5339, Facsimile +64-4-463-5331

Email Biosci@vuw.ac.nz 


\section{VICTORIA UNIVERSITY OF WELLINGTON \\ Te Whare Wānanga o te Ūpoko o te Ika a Māui}

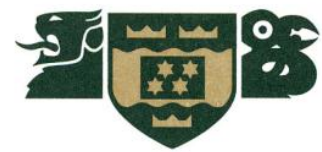

Urban wildlife management and feral pigeons in Wellington

$15^{\text {th }}$ November 2010

Researcher: Symone Krimowa

Telephone (mobile): 0211408967

Email: symonekrimowa@gmail.com
Supervisor: Dr Wayne Linklater

Telephone: 044635233

Email: wayne.linklater@vuw.ac.nz
School of Biological Sciences

Victoria University of Wellington

PO Box 600

Wellington 6140

Dear Owner/Manager,

Recently a questionnaire about the effects of feral pigeons on businesses in Wellington was mailed to you. Your business was chosen due to its location in Wellington. We have received many questionnaires from other businesses and yours will also make an important contribution.

If you have already completed and returned the two-page questionnaire, please accept my sincere thanks. If not, it would be much appreciated if you could do so and will only take up to ten minutes to complete. A copy of the survey can also be found online at http://www.victoria.ac.nz/sbs/staff/wayne-linklater.aspx.

I'm especially grateful for your help because it is only by asking people like you to share your knowledge that we can investigate bird-related knowledge and behaviour. The study is an important one as it will help establish the relationship between people and wildlife in cities, and provide lessons for urban wildlife management.

Thank you very much for helping with this important study.

Sincerely,

Symone Krimowa

MSc candidate, Victoria University of Wellington

\section{SCHOOL OF BIOLOGICAL SCIENCES}

Te Kura Mātauranga Koiora

FACULTY OF SCIENCE

Te Wāhanga Pūtaiao

P.O. Box 600, Wellington, New Zealand

Telephone +64-4-463-5339, Facsimile +64-4-463-5331

Email Biosci@vuw.ac.nz 


\section{Bibliography}

Adams, C., Lindsey, K., Ash, S., 2006. Urban wildlife management. CRC Press.

Anderies, J., Katti, M., Shochat, E., 2007. Living in the city: Resource availability, predation, and bird population dynamics in urban areas. Journal of theoretical biology 247 (1), 36-49.

Arcese, P., Smith, J., 1988. Effects of population density and supplemental food on reproduction in song sparrows. The Journal of Animal Ecology, 119-136.

Balram, S., Dragievi, S., 2005. Attitudes toward urban green spaces: integrating questionnaire survey and collaborative gis techniques to improve attitude measurements. Landscape and Urban Planning 71 (2-4), $147-162$.

Boutin, S., 1990. Food supplementation experiments with terrestrial vertebrates: patterns, problems, and the future. Canadian Journal of Zoology 68 (2), 203-220. 
Brown, M., 1996. Assessing body condition in birds. Curr Ornithol 13, 67121.

Buijs, J. A., Wijnen, J. H. V., 2001. Survey of feral rock doves (columba livia) in amsterdam, a bird-human association. Urban Ecosystems 5, 235-241.

Burnham, K., Anderson, D., 2002. Model selection and multimodel inference: a practical information-theoretic approach. Springer Verlag.

Caula, S., Hvenegaard, G., Marty, P., 2009. The influence of bird information, attitudes, and demographics on public preferences toward urban green spaces: The case of montpellier, france. Urban Forestry \& Urban Greening 8 (2), 117-128.

Ciminari, M., Moyano, G., Chediack, J., Caviedes-Vidal, E., 2005. Feral pigeons in urban environments: dietary flexibility and enzymatic digestion. Revista Chilena de Historia Natural 78, 267-279.

Clark, H., 2011. Urban carnivores: Ecology, conflict, and conservation. Western North American Naturalist 70 (4), 577-578.

Cordano, M., Frieze, I., Ellis, K., 2004. Entangled affiliations and attitudes: an analysis of the influences on environmental policy stakeholders' behavioral intentions. Journal of Business Ethics 49 (1), 27-40.

Council, W. C., 2010.

URL www. wellington.govt.nz

Dilks, P. J., 1975. The breeding of the feral pigeon (columba livia) in hawke's bay new zealand. Notornis 22, 295-301. 
Ditchkoff, S. S., Saalfeld, S. T., Gibson, C. J., 2006. Animal behavior in urban ecosystems: Modifications due to human-induced stress. Urban Ecosystems 9, 5-12.

Dobeic, M., 2003. Regulation of population size of street pigeons in ljubljana, slovenia. Acta veterinaria 53 (2-3), 171-182.

DominionPost, 2008. Pigeon cull called off.

Dorzhiev, T., 1991. Ecology of Sympatric Pigeon Populations. Moscow: Nauka.

Dowding, J., Murphy, E., 2001. The impact of predation by introduced mammals on endemic shorebirds in new zealand: a conservation perspective. Biological Conservation 99 (1), 47-64.

Dunn, R. R., Gavin, M. C., Sanchez, M., Solomon, J. N., 2006. The pigeon paradox: dependence of global conservation on urban nature. Conservation Biology 20 (6), 1814-1816.

Eagly, A., Chaiken, S., 1993. The psychology of attitudes. Harcourt Brace Jovanovich College Publishers.

Ericsson, G., Heberlein, T., 2003. Attitudes of hunters, locals, and the general public in sweden now that the wolves are back. Biological conservation 111 (2), 149-159.

Fazio, R., 1986. How do attitudes guide behavior. Handbook of motivation and cognition: Foundations for social behavior 1, 204-233. 
Fazio, R., Zanna, M., 1981. Direct experience and attitude-behavior consistency. Advances in experimental social psychology 14, 161-202.

Fransson, N., Gärling, T., 1999. Environmental concern: Conceptual definitions, measurement methods, and research findings. Journal of environmental psychology 19 (4), 369-382.

Google, November 2010. Map of wellington.

URL www.maps.google.com

Haag-Wackernagel, D., 1995. Regulation of the street pigeon in basel. Wildlife Society Bulletin 23 (2), 256-260.

Haag-Wackernagel, D., Geigenfeind, I., 2008. Protecting buildings against feral pigeons. Eur J Wildl Res 54, 715-721.

Haag-Wackernagel, D., Heeb, P., Leiss, A., 2006. Phenotype-dependent selection of juvenile urban feral pigeons columba livia: Capsule we suggest the existence of colour-based selection processes on juvenile feral pigeons in an urban environment. Bird Study 53 (2), 163-170.

Haag-Wackernagel, D., Moch, H., 2004. Health hazards posed by feral pigeons. J Inf 48(4), 307-313.

Henriques, I., Sadorsky, P., 1999. The relationship between environmental commitment and managerial perceptions of stakeholder importance. Academy of management Journal, 87-99.

Jerolmack, C., 2008. How pigeons became rats: The cultural-spatial logic of problem animals. Social Problems 55 (1), 72-94. 
Johnson, D., 1980. The comparison of usage and availability measurements for evaluating resource preference. Ecology 61 (1), 65-71.

Johnson, J., Omland, K., 2004. Model selection in ecology and evolution. Trends in Ecology \& Evolution 19 (2), 101-108.

Johnson, M., Brace, P., Arceneaux, K., 2005. Public opinion and dynamic representation in the american states: The case of environmental attitudes*. Social Science Quarterly 86 (1), 87-108.

Johnston, R. F., Janiga, M., 1995. Feral Pigeons. Oxford University Press.

Jokimäki, J., Suhonen, J., 1998. Distribution and habitat selection of wintering birds in urban environments. Landscape and Urban Planning 39 (4), 253-263.

Keating, K., Cherry, S., 2004. Use and interpretation of logistic regression in habitat-selection studies. Journal of Wildlife Management 68 (4), 774789.

Krebs, L., 1974. Feral pigeon control. In: Proceedings of the 6th Vertebrate Pest Conference (1974). p. 26.

Lancaster, R., Rees, W., 1979. Bird communities and the structure of urban habitats. Canadian Journal of Zoology 57 (12), 2358-2368.

Lefebvre, L., 1983. Equilibrium distribution of feral pigeons at multiple food resources. Behavioral Ecology and Sociobiology 12, 11-17. 
Luniak, M., 2004. Synurbization-adaptation of animal wildlife to urban development. In: Proceedings of the 4 th International Symposium on Urban Wildlife Conservation. Tuscon (AZ): University of Arizona. Citeseer, pp. 50-55.

Madden, F., 2004. Creating coexistence between humans and wildlife: Global perspectives on local efforts to address human-wildlife conflict. Human Dimensions of Wildlife 9, 247-257.

Major, R., Gowing, G., Kendal, C., 1996. Nest predation in australian urban environments and the role of the pied currawong, strepera graculina. Australian Journal of Ecology 21 (4), 399-409.

Manly, B., 2002. Resource selection by animals: statistical design and analysis for field studies. Springer.

Miskelly, C., Dowding, J., Elliott, G., Hitchmough, R., Powlesland, R., Robertson, H., Sagar, P., Scofield, R., Taylor, G., 2008. Conservation status of new zealand birds, 2008. Notornis 55 (3), 117-135.

Murton, R., Thearle, R., Thompson, J., 1972a. Ecological studies of the feral pigeon columba livia var. i. population, breeding biology and methods of control. Journal of Applied Ecology 9 (3), 835-874.

Murton, R., Westwood, N., 1966. The foods of the rock dove and feral pigeon. Bird Study 13 (2), 130-146.

Murton, R. K., Coombs, C. F. B., Thearle, R. J. P., Dec 1972b. Ecological 
studies of the feral pigeon columba livia var. ii flock behaviour and social organization. Journal of Applied Ecology 9 (3), 875-889.

Nam, D., Lee, D., Koo, T., 2004. Factors causing variations of lead and cadmium accumulation of feral pigeons (columba livia). Environmental monitoring and assessment 95 (1), 23-35.

Neu, C., Byers, C., Peek, J., 1974. A technique for analysis of utilizationavailability data. The Journal of Wildlife Management 38 (3), 541-545.

Newton, I., 1980. The role of food in limiting bird numbers. Ardea 68 (1), $11-30$.

Owen, M., Cook, W., 1977. Variations in body weight, wing length and condition of mallard anas platyrhynchos platyrhynchos and their relationship to environmental changes. Journal of Zoology 183 (3), 377-395.

Palameta, B., Lefebvre, L., 1985. The social transmission of a food-finding technique in pigeons: what is learned? Animal Behaviour 33 (3), 892 896.

Patterson, M. E., Montag, J. M., Williams, D. R., 2003. The urbanization of wildlife management: social science, conflict, and decision-making. Urban Forestry and Urban Greening 1, 171-183.

Posada, D., Buckley, T., 2004. Model selection and model averaging in phylogenetics: advantages of akaike information criterion and bayesian approaches over likelihood ratio tests. Systematic Biology 53 (5), 793. 
Rebele, F., 1994. Urban ecology and special features of urban ecosystems. Global ecology and biogeography letters 4 (6), 173-187.

Reiter, D. K., Brunson, M. W., Schmidt, R. H., 1999. Public attitudes toward wildlife damage management and policy. Wildlife Society Bulletin 27 (3), 746-758.

Robertson, C., Fraser, M., Pickard, C., 2007. Atlas of Bird Distribution in New Zealand. Ornithological Society of New Zealand.

Rollinson, D., O'Leary, R., Jones, D. N., 2003. The practice of wildlife feeding in suburbane brisbane. Corella 27, 52-58.

Rose, E., Haag-Wackernagel, D., Nagel, P., 2006. Practical use of gpslocalization of feral pigeons columba livia in the urban environment. Ibis $148(2), 231-239$.

Ryan, A., 2011. The distribution, density and movements of feral pigeons columba livia and their relationship with people. Master's thesis, School of Biological Sciences, Victoria University of Wellington.

Sacchi, R., Gentilli, A., Razzetti, E., Barbieri, F., 2002. Effects of building features on density and flock distribution of feral pigeons columba livia var. domestica in an urban environment. Canadian Journal of Zoology $80,48-54$.

Schein, M., 1954. Survival records of young feral pigeons. The Auk 71 (3), 318-320. 
Schelly, C., Cross, J., Franzen, W., Hall, P., Reeve, S., 2011. Reducing energy consumption and creating a conservation culture in organizations: A case study of one public school district. Environment and Behavior $43(3), 316$.

Shochat, E., 2004. Credit or debit? resource input changes population dynamics of city-slicker birds. Oikos 106 (3), 622-626.

Shochat, E., Lerman, S., Katti, M., Lewis, D., 2004. Linking optimal foraging behavior to bird community structure in an urban-desert landscape: field experiments with artificial food patches. American Naturalist $164(2), 232-243$.

Sillero-Zubiri, C., Sukumar, R., Treves, A., 2007. Living with wildlife: the roots of conflict and the solutions. Key topics in conservation biology, $266-272$.

Smith, J., Montgomerie, R., Taitt, M., Yom-Tov, Y., 1980. A winter feeding experiment on an island song sparrow population. Oecologia 47 (2), 164-170.

Sol, D., Santos, D., Garcia, J., Cuadrado, M., 1998. Competition for food in urban pigeons: the cost of being juvenile. The Condor 100 (2), 298-304.

Sol, D., Senar, J., 1995. Urban pigeon populations: stability, home range, and the effect of removing individuals. Canadian journal of zoology 73 (6), 1154-1160. 
Sorace, A., 2002. High density of bird and pest species in urban habitats and the role of predator abundance. Ornis Fennica 79 (2), 60-71.

Statistics, N. Z., 2006.

Stern, P., Kalof, L., Dietz, T., Guagnano, G., 1995. Values, beliefs, and proenvironmental action: Attitude formation toward emergent attitude objects. Journal of Applied Social Psychology 25 (18), 1611-1636.

Straughan, R., Roberts, J., 1999. Environmental segmentation alternatives: a look at green consumer behavior in the new millennium. Journal of consumer Marketing 16 (6), 558-575.

Sutherland, W., Newton, I., Green, R., 2004. Bird ecology and conservation: a handbook of techniques. Oxford University Press, USA.

Tangley, L., 1986. The urban ecologist. BioScience 36(2), 68-71.

Temby, I., 2004. Urban wildlife issues in australia. In: 4th International Urban Wildlife Symposium, University of Arizona, Tuscon.

TheAge, February 2007. You can lead a pigeon to the yarra, but you cannot make it nest.

Tsurim, I., Abramsky, Z., Kotler, B. P., 2008. Foraging behavior of urban birds: are human commensals less sensitive to predation risk than their nonurban counterparts? The Condor 110 (4), 772-776.

UnitedNations, 2009. World urbanization prospects: The 2009 revision. 
Tech. rep., United Nations.

URL http://esa.un.org/unpd/wup/index.htm

Valcarcel, A., Fernández-Juricic, E., 2009. Antipredator strategies of house finches: are urban habitats safe spots from predators even when humans are around? Behavioral Ecology and Sociobiology 63 (5), 673-685.

Vorkinn, M., Riese, H., 2001. Environmental concern in a local context. Environment and Behavior 33 (2), 249-263.

Walker, S., Price, R., Rutledge, D., Stephens, R., Lee, W., 2006. Recent loss of indigenous cover in new zealand. New Zealand Journal of Ecology $30(2), 169-177$.

Warren, P., Katti, M., Ermann, M., Brazel, A., 2006. Urban bioacoustics: it's not just noise. Animal Behaviour 71 (3), 491-502.

YellowPages, 2010. Wellington Yellow Pages, print Edition. Yellow Pages Group. 